\title{
Two Interacting Coordinate Hopf Algebras of Affine Groups of Formal Series on a Category
}

\author{
Laurent Poinsot \\ Université Paris 13, Sorbonne Paris Cité, LIPN, CNRS (UMR 7030), 93430 Villetaneuse, France \\ Correspondence should be addressed to Laurent Poinsot; laurent.poinsot@lipn.univ-paris13.fr
}

Received 19 March 2013; Revised 17 May 2013; Accepted 14 July 2013

Academic Editor: Stefaan Caenepeel

Copyright (C) 2013 Laurent Poinsot. This is an open access article distributed under the Creative Commons Attribution License, which permits unrestricted use, distribution, and reproduction in any medium, provided the original work is properly cited.

\begin{abstract}
A locally finite category is defined as a category in which every arrow admits only finitely many different ways to be factorized by composable arrows. The large algebra of such categories over some fields may be defined, and with it a group of invertible series (under multiplication). For certain particular locally finite categories, a substitution operation, generalizing the usual substitution of formal power series, may be defined, and with it a group of reversible series (invertible under substitution). Moreover, both groups are actually affine groups. In this contribution, we introduce their coordinate Hopf algebras which are both free as commutative algebras. The semidirect product structure obtained from the action of reversible series on invertible series by anti-automorphisms gives rise to an interaction at the level of their coordinate Hopf algebras under the form of a smash coproduct.
\end{abstract}

\section{Introduction}

The set $G$ of formal power series in one variable $x$, such as, $1+x T$, where $T \in R[[x]]$, forms a group under the usual multiplication of series (whenever $R$ is a commutative ring with a unit). Moreover, the set $H$ of series, such as, $x+x^{2} T$, $T \in R[[x]]$, forms a group under another operation, namely, the substitution. For any $S=\sum_{n \geq 0} s_{n} x^{n} \in R[[x]]$, and $T \in H$, the substitution of $S$ by $T$ is defined as the series $\sum_{n \geq 0} s_{n} T^{n}$ (the fact that $T$ begins with $x$ implies that $\left(T^{n}\right)_{n \geq 0}$ is summable in the usual topology of series). This actually gives rise to a semidirect product of groups $G \rtimes H^{\mathrm{op}}$ (where $H^{\mathrm{op}}$ is the opposite group of $H$ ). Actually, this situation may be generalized in the following way. Let $C$ be a category in which any arrow admits only finitely many factorizations by composable arrows. Such a category is referred to as a locally finite category. A locally finite category admits a large algebra, that is, the set of all set-theoretic maps from the arrows of the category to some base (commutative) ring may be multiplied by a Cauchy-kind product inherited from the composition of arrows in the category. Now, the set $\mathfrak{I}$ of all series in this large algebra with a coefficient 1 at each identity arrow in the category forms a group under multiplication. Moreover, given a finite semicategory $C$, roughly speaking a category without identities, we may construct the free category $\underline{C}^{*}$ over the underlying graph structure of $C$, which is a locally finite category. According to a universal property, we may define in a unique way an evaluation functor that maps formal nonvoid paths in $C^{*}$ (nonvoid sequences of composable arrows in $C$ ) to the result in $C$ of their compositions. This gives rise to an operation of substitution on the large algebra of $C^{*}$ similar to the substitution of formal power series. The set $\mathfrak{M}_{1}$ of all series in the large algebra which are zero on the identity arrows and 1 on the formal paths of length one (the arrows of C) forms a group under substitution. Parallelizing the usual situation of formal power series, it appears that this group acts by anti-automorphisms on the group $\mathfrak{\Im}$ and therefore defines a semidirect product $\mathfrak{I} \rtimes \mathfrak{M}_{1}^{\text {op }}$. Moreover both groups $\mathfrak{I}$ and $\mathfrak{M}_{1}$ are actually affine groups and so admit coordinate Hopf algebras which appear to be free as commutative algebras. In this contribution, we present the constructions of both affine groups $\mathfrak{I}$ and $\mathfrak{M}_{1}$ and introduce their coordinate Hopf algebras which may be thought as generalizing some wellknown Hopf algebras.

\section{Basic Definitions and Notations}

In this paper, $R$ denotes a commutative ring with a unit. In general, $R$-algebras are not assumed to possess a unit nor 
to be commutative but they are associative. The notation $R$-CAlg stands for the category of commutative $R$-algebras with a unit and unit preserving algebra maps, and the homsets are denoted by $R-\operatorname{CAlg}(A, B)$. In what follows, if $A$ is $R$ algebra with a unit, then $1_{A}$ is its identity.

Let $X$ be any set, and let $f \in R^{X}$. The support of $f$ is the set $\{x \in X: f(x) \neq 0\}$. Such a map $f$ is said to be finitely supported or has a finite support when its support is a finite set. The set of all finitely supported maps from $X$ to $R$ is denoted by $R^{(X)}$. It is free as a module with basis $\left\{\delta_{x}: x \in X\right\}$ where $\delta_{x}$ is the map such that $\delta_{x}(y)=0$ if $y \neq x$ and $\delta_{x}(x)=1$. In what follows, we identify $x \in X$ with its image $\delta_{x}$ in $R^{(X)}$ so that any map $p \in R^{(X)}$ may be written in a unique way as a linear combination $\sum_{x \in X} p(x) x$. The module $R^{(X)}$ is actually a submodule of the product $R^{X}$. There is a duality bracket between $R^{X}$ and $R^{(X)}$, namely, the $R$-bilinear form $\langle\cdot \mid \cdot\rangle: R^{X} \times R^{(X)} \rightarrow R$ such that for every $f \in R^{X}, p \in R^{(X)}$,

$$
\langle f \mid p\rangle=\sum_{x \in X} f(x) p(x)
$$

(the sum has only finitely many nonzero terms because $p$ is finitely supported). It is obviously a two-sided nondegenerate. In particular, for every $f \in R^{X}$ and every $x \in X, f(x)=\langle f|$ $x\rangle$, and for every $p \in R^{(X)}, p=\sum_{x \in X}\langle p \mid x\rangle x$. We observe that for each $x \in X, \delta_{x}$ may be identified with an element of $\left(R^{X}\right)^{*}$ through $\langle\cdot \mid \cdot\rangle$, namely, $\delta_{x}(f)=\langle f \mid x\rangle=f(x)$ for every $f \in R^{X}$.

When $R$ is equipped with the discrete topology, and $R^{X}$ has the product topology, then it becomes a (Hausdorff) complete topological module (the addition and $f \mapsto-f$ are continuous, and scalar multiplication is jointly continuous), and it is the completion of the topological $R$-module $R^{(X)}$ (equipped with the product topology inherited from that of $\left.R^{X}\right)$. Moreover, for any $f \in R^{X}$, the family $(\langle f \mid x\rangle x)_{x \in X}$ is summable (see [1]) in $R^{X}$ with sum $f$ so that we may represent $f$ as an infinite linear combination $f=\sum_{x \in X}\langle f \mid x\rangle x$ (more details may be found in [2-4]). Moreover, $\langle\cdot \mid \cdot\rangle$ is separately continuous.

\section{A General Approach on Coordinate Hopf Algebras of a Group of Series}

In this section, we present in a general way the notion of coordinate Hopf algebra on a group-valued functor of $A$ valued functions defined on some set $Z$, for varying algebras $A$. The result presented here will be used in the sequel to define two coordinate Hopf algebras of two groups of formal series that define a semidirect product. The reader should refer to $[5,6]$ for basic definitions about Hopf algebras, to [79] for the notions concerning algebraic groups, and to [10] for category-theoretic concepts.

Let $A$ be a commutative $R$-algebra with a unit, where $R$ is a commutative ring with unit. An R-group is a functor from $R$-CAlg to the category of groups Grp. When an $R$ group $G$ is representable when viewed as a set-valued functor (by composition with the forgetful functor from Grp to the category of sets Set), that is, $G(B) \cong R-\operatorname{CAlg}(A, B)$ (isomorphic as sets, natural in $B$ ) for some commutative $R$-algebra $A$, then it is called an affine group (or proaffine algebraic group when the base ring $R$ is a field), and $A$ (determined up to a unique isomorphism) is referred to as the coordinate Hopf algebra $\mathcal{O}(G)$ of $G$, for reasons made clear hereafter. The representable $R$-group $G$ is an affine algebraic group when $\mathcal{O}(G)$ is finitely generated as an $R$-algebra. Since the multiplication $m_{B}: G(B) \times G(B) \rightarrow G(B)$, the inversion $i_{B}: G(B) \rightarrow G(B)$, and the unit element $e_{B}: \star \rightarrow$ $G(B)$ are natural transformations between representable settheoretic functors, by Yoneda's lemma they uniquely give rise respectively to a (coassociative) coproduct $\Delta: \mathcal{O}(G) \rightarrow$ $\mathcal{O}(G) \otimes_{R} \mathcal{O}(G)$, an antipode $S: \mathcal{O}(G) \rightarrow \mathcal{O}(G)$, and a counit $\mathcal{O}(G) \rightarrow R$ that turn the algebra $\mathcal{O}(G)$ into a commutative Hopf $R$-algebra. It turns that the natural set isomorphisms $G(B) \cong R-\operatorname{CAlg}(O(G), B)$ become group isomorphisms.

In what follows we will be interested in the following situation. Let $G$ be an $R$-group, and let $Z$ be a set. We assume that there exists a subset $X$ of $Z$ such that, as a set-valued functor, $G$ is isomorphic to $\operatorname{Set}(X, U(\cdot))$, where $U: R$ $\mathrm{CAlg} \rightarrow$ Set is the forgetful functor, that is, for every algebra $A, \alpha_{A}: G(A) \cong A^{X} \subseteq A^{Z}$ (bijection natural in $A$ ), and for every algebra map $f: A \rightarrow B, \alpha_{B}(G(f)(S))=f \circ \alpha_{A}(S)$ for each $S \in G(A)$. This is equivalent to say that $G$ is represented by the free commutative $R$-algebra $R[X]$. In this situation, we say that $X$ is the coordinate system of $G$ (obviously when it exists, $X$ is uniquely determined up to bijections). We may view any $S \in A^{X}$ as a formal series $\sum_{x \in X}\langle S \mid x\rangle x$. The functor $G \times G$ is represented by the algebra $R[X] \otimes_{R} R[X]$, while $R$ represents the constant functor $*$ equal to a onepoint set. By Yoneda's lemma, the multiplication $m: G \times$ $G \rightarrow G$ uniquely determines an algebra map $\Delta \epsilon$ $\left(R[X] \otimes_{R} R[X]\right)^{X} \cong R-\operatorname{CAlg}\left(R[X], R[X] \otimes_{R} R[X]\right)$ which is explicitly given by $\alpha_{R[X] \otimes_{R} R[X]}\left(m_{R[X] \otimes_{R} R[X]}\left(\alpha_{R[X] \otimes_{R} R[X]}^{-1}(S)\right.\right.$, $\left.\left.\alpha_{R[X] \otimes_{R} R[X]}^{-1}(T)\right)\right)$ where $S=\sum_{x \in X}(x \otimes 1) x \in R[X]^{X}$ and $T=\sum_{x \in X}(1 \otimes x) x \in R[X]^{X}$ (essentially because $\operatorname{id}_{R[X] \otimes_{R} R[X]}$ is induced by the pair of maps $(S, T)$ under the isomorphism $R-C A l g\left(R[X] \otimes_{R} R[X], R[X] \otimes_{R} R[X]\right) \cong\left(R[X] \otimes_{R} R[X]\right)^{X} \times$ $\left.\left(R[X] \otimes_{R} R[X]\right)^{X}\right)$. Similarly, again by Yoneda's lemma, the unit $e: * \rightarrow G$ uniquely determines an algebra map $\epsilon:$ $R[X] \rightarrow R$ given by $\epsilon=\alpha_{R}\left(1_{G(R)}\right) \in R^{X}$ (with $1_{G}$ the identity of a group $G$ ). Finally, the inversion $i: G \rightarrow G$ gives rise to a unique algebra map $s \in R[X]^{X}$ explicitly given by $s=\alpha_{R[X]}\left(i_{R[X]}\left(\alpha_{R[X]}^{-1}\left(\sum_{x \in X} x x\right)\right)\right)$, where $\sum_{x \in X} x x$ is the member $R[X]^{X}$ that induces the identity $\operatorname{id}_{R[X]}$. This map $s$ is of course the antipode of $R[X]$.

In what follows we introduce two affine groups $\mathfrak{\Im}$ and $\mathfrak{M}_{1}$ (see Section 6), whose $R$-rational points are groups of formal series on some locally finite categories, and whose coordinate rings are both free commutative algebras (as in the aforementional discussion). As $R$-groups, they interact under the form of an $R$-group semidirect product (a semidirect product natural in its algebra variable). The main result of this paper is the following. 
Theorem 1. Let $\mathbb{K}$ be a field. The $\mathbb{K}$-group $\mathfrak{\Im} \rtimes \mathfrak{M}_{1}^{\mathrm{op}}$ is actually an affine group.

Example 2. Let $x$ be a variable, $R$ a commutative ring with unit, and $A$ a commutative $R$-algebra with a unit. We present two examples of coordinate Hopf algebras which are well known (see [11-13]) and that are generalized hereafter.

(1) Let $G$ be the $R$-group of invertible formal power series $G(A)=\{S \in A[[x]]:\langle S \mid 1\rangle=1\}$, that is, $S \in G(A)$, if, and only if, $S=1+x T$, where $T \in A[[x]]$. Let $\left(x_{n}\right)_{n \geq 1}$ be an infinite sequence of pairwise distinct variables. Let us compute the coproduct $\Delta$, counit $\epsilon$, and antipode $s$ of free commutative algebra $R\left[x_{n}\right.$ : $n \geq 1$ ]. According to the previous discussion, $\Delta$ is obtained by computing the product in $G\left(R\left[x_{n}: n \geq\right.\right.$ 1] $\left.\otimes_{R} R\left[x_{n}: n \geq 1\right]\right)$ of the series $S=1+\sum_{n \geq 1}\left(x_{n} \otimes\right.$ 1) $x^{n}$ and $T=1+\sum_{n \geq 1}\left(1 \otimes x_{n}\right) x^{n}$ which is equal to $1+\sum_{n \geq 1}\left(x_{n} \otimes 1+1 \otimes x_{n}+\sum_{i+j=n ; i, j \geq 1}\left(x_{i} \otimes x_{j}\right)\right) x^{n}$. Then, $\Delta=\sum_{n \geq 1}\left(x_{n} \otimes 1+1 \otimes x_{n}+\sum_{i+j=n ; i, j \geq 1} x_{i} \otimes x_{j}\right) x^{n}$ so that for every $n \geq 1, \Delta\left(x_{n}\right)=x_{n} \otimes 1+1 \otimes x_{n}+$ $\sum_{i+j=n ; i, j \geq 1} x_{i} \otimes x_{j}$. The counit is given by the settheoretic map $\epsilon\left(x_{n}\right)=0$ for each $n \geq 1$. The inverse of $S \in G(A)$ is given recursively by $\left\langle S^{-1} \mid 1\right\rangle=1$, and $\left\langle S^{-1} \mid x^{n+1}\right\rangle=-\left\langle S \mid x^{n+1}\right\rangle-\sum_{i+j=n+1 ; i, j \geq 1}\langle S|$ $\left.x^{i}\right\rangle\left\langle S^{-1} \mid x^{j}\right\rangle$ for all $n \geq 0$. In particular, for $S=$ $1+\sum_{n \geq 1} x_{n} x^{n} \in G\left(R\left[x_{n}: n \geq 1\right]\right)$, for each $n \geq 1$, $\left\langle S^{-1} \mid x^{n}\right\rangle=-x_{n}-\sum_{i=1}^{n-1} x_{i}\left\langle S^{-1} \mid x^{n-i}\right\rangle$. It follows that $s=\sum_{n \geq 1}\left\langle S^{-1} \mid x^{n}\right\rangle x_{n}$ or in other terms $s\left(x_{n}\right)=$ $-x_{n}+\sum_{i=1}^{n-1} x_{i} s\left(x_{n-i}\right)$ for all $n \geq 1$.

(2) Let $H(A)$ be the set $\{S \in A[[x]]:\langle S \mid 1\rangle=$ $\left.0,\langle S \mid x\rangle=1_{A}\right\}$, that is, $S \in H(A)$, if, and only if, $S=x+x^{2} T$ for $T \in A[[x]]$. This forms a group under the usual composition of formal power series $S \circ T=x+\sum_{n>2}\left\langle S \mid x^{n}\right\rangle T^{n}$. It is clear that $\left\{x_{n}\right.$ : $n \geq 2\}$ is the coordinate system of $H$. Let us explicitly compute the bialgebra structure of the corresponding coordinate Hopf algebra $R\left[x_{n}: n \geq 2\right]$. Following [14] (or for instance by the usual Faà di Bruno formula, see $[15,16])$, we have $\left\langle S \circ T \mid x^{2}\right\rangle=\left\langle S \mid x^{2}\right\rangle+\left\langle T \mid x^{2}\right\rangle$ and $\left\langle S \circ T x^{n+1}\right\rangle=\left\langle S \mid x^{n+1}\right\rangle+\left\langle T \mid x^{n+1}\right\rangle+$ $\sum_{k=2}^{n}\left\langle S \mid x^{k}\right\rangle B_{k}\left(\left\langle T \mid x^{2}\right\rangle, \ldots,\left\langle T \mid x^{k}\right\rangle\right)$, where $B_{k}$ is a polynomial in $k-1$ variables. Applying this to $S=x+\sum_{n \geq 2}\left(x_{n} \otimes 1\right) x^{n}$ and $T=x+\sum_{n \geq 2}\left(1 \otimes x_{n}\right) x^{n}$ gives the coproduct $\Delta=\sum_{n \geq 2}\left(x_{n} \otimes 1+1 \otimes x_{n}+\sum_{k=2}^{n-1}\left(x_{k} \otimes\right.\right.$ $\left.1) B_{k}\left(1 \otimes x_{2}, \ldots, 1 \otimes x_{k}\right)\right) x_{n}$ or equivalently, $\Delta\left(x_{n}\right)=$ $x_{n} \otimes 1+1 \otimes x_{n}+\sum_{k=2}^{n-1}\left(x_{k} \otimes 1\right) B_{k}\left(1 \otimes x_{2}, \ldots, 1 \otimes x_{k}\right)$ for each $n \geq 2$. The counit is given by $\epsilon\left(x_{n}\right)=0$ for each $n \geq 2$. Let $S \in H(A)$ and $T$ be the inverse of $S$ in $H(A)$. For each $n \geq 2$, we have $0=\left\langle S \circ T \mid x^{n}\right\rangle=\sum_{k=1}^{n}\langle S|$ $\left.x^{k}\right\rangle \sum_{i_{1}+\cdots+i_{k}=n}\left\langle T \mid x^{i_{1}}\right\rangle \cdots\left\langle T \mid x^{i_{k}}\right\rangle=\left\langle T \mid x^{n}\right\rangle+$ $\sum_{k=2}^{n}\left\langle S \mid x^{k}\right\rangle \sum_{i_{1}+\cdots+i_{k}=n}\left\langle T \mid x^{i_{1}}\right\rangle \cdots\left\langle T \mid x^{i_{k}}\right\rangle$. Applying this formula to $S=x+\sum_{n \geq 2} x_{n} x^{n} \in H\left(R\left[x_{n}: n \geq\right.\right.$ 2]) gives $s\left(x_{n}\right)=-\sum_{k=2}^{n} x_{k} \sum_{i_{1}+\cdots+i_{k}=n} s\left(x_{i_{1}}\right) \cdots s\left(x_{i_{k}}\right)$ (where by convention we put $s\left(x_{0}\right) \stackrel{2}{=}, s\left(x_{1}\right)=1$ ) for each $n \geq 2$.

\section{Categories, Semicategories, and Their Total Algebra}

The basic concepts from category theory may be found in [10] but are recalled hereafter. When viewed as algebraic objects, the categories are always considered as small categories in the sense that their classes of objects and arrows form usual sets (in some given universe). The reader should refer to [10] for this kind of size issues.

A semicategory, also called a taxonomy, see [17], (respectively, category) $C$ is given by its class of objects $\mathrm{Ob}(C)$, its class of arrows or morphisms $\operatorname{Arr}(C)$, two (non necessarily surjective) maps $\partial_{0}, \partial_{1}: \operatorname{Arr}(C) \rightarrow \mathrm{Ob}(C)$ (and $\iota: \mathrm{Ob}(C) \rightarrow$ $\operatorname{Arr}(C)$ such that $\partial_{j} \circ \iota=\operatorname{id}_{0 \mathrm{~b}(C)}, j=1,2$, if $C$ is a category), $\partial_{0}$ is the domain map, $\partial_{1}$ is the codomain map, (and $\iota$ is the identity arrow map, if $C$ is a category), and finally a map, called composition, $\gamma: \operatorname{Arr}(C) \times{ }_{\mathrm{ob}(C)} \operatorname{Arr}(C)=$ $\left\{(f, g): \operatorname{Arr}(C)^{2}: \partial_{0}(f)=\partial_{1}(g)\right\} \rightarrow \operatorname{Arr}(C)$ such that $\partial_{0}(\gamma(f, g))=\partial_{0}(g)$ and $\partial_{1}(\gamma(f, g))=\partial_{1}(f)$ for all $(f, g) \in \operatorname{Arr}(C) \times{ }_{\mathrm{ob}(C)} \operatorname{Arr}(C)$, and that satisfies an axiom of associativity: $\gamma(f, \gamma(g, h))=\gamma(\gamma(f, g), h)$ for all $f, g, h \in$ $\operatorname{Arr}(C)$ such that $\partial_{0}(f)=\partial_{1}(g)$ and $\partial_{0}(g)=\partial_{1}(h)$, (and two axioms of identity: $\gamma\left(f, \iota\left(\partial_{0}(f)\right)\right)=f$ and $\gamma\left(\iota\left(\partial_{1}(f)\right), f\right)=f$ for every $f \in \operatorname{Arr}(C)$, if $C$ is a category). In general, $\gamma$ is denoted by $\circ$ and $\gamma(f, g)=f \circ g$, and $\iota(x)$ by id $_{x}$ for each $x \in \mathrm{Ob}(C)$. The set $\operatorname{Arr}(C) \times_{\mathrm{ob}(C)} \operatorname{Arr}(C)$ is called the set of composable arrows in $C$. More generally, for every $n \geq 2$, $\underbrace{\operatorname{Arr}(C) \times_{\mathrm{ob}(C)} \cdots \times_{\mathrm{ob}(C)} \operatorname{Arr}(C)}=\left\{\left(f_{1}, \ldots, f_{n}\right) \in \operatorname{Arr}(C)^{n}:\right.$

$\left.\partial_{1}\left(f_{i+1}\right)={ }^{n \text { factors }} \partial_{0}\left(f_{i}\right), i=1, \ldots, n-1\right\}$ is the set of $n$-tuple of composable arrows, and by associativity, $f_{1} \circ \cdots \circ f_{n}$ has an obvious meaning whenever $\left(f_{1}, \ldots, f_{n}\right)$ is an $n$-tuple of composable arrows. It is also clear that in this case $\partial_{0}\left(f_{1}\right.$ 。 $\left.\cdots \circ f_{n}\right)=\partial_{0}\left(f_{n}\right)$ and $\partial_{1}\left(f_{1} \circ \cdots \circ f_{n}\right)=\partial_{1}\left(f_{1}\right)$. We may also denote by $f: a_{0} \rightarrow a_{1}$ or by $f \in C\left(a_{0}, a_{1}\right)$ the fact that $f \in \operatorname{Arr}(C)$ with $\partial_{j}(f)=a_{j} \in \mathrm{Ob}(C), j=0,1$. Functors between semicategories are the obvious ones.

Remark 3. If $C$ is a semicategory, then there is the possibility that some objects do not correspond to domain or codomain of arrows (this is a kind of isolated point in a graph), because the map $\partial_{j}$ is not assumed to be onto, while it is for a category.

Let $R$ be any commutative ring with a unit, and let $A$ be a commutative $R$-algebra with a unit. We may define the $A$ algebra of $C$, denoted by $A\langle C\rangle$, as the free $A$-module with basis $\operatorname{Arr}(C)$ together with the following constants of structure (see [18]) that define an associative product:

$$
f \times g= \begin{cases}f \circ g & \text { if }(f, g) \in \operatorname{Arr}(C) \times \times_{\mathrm{ob}(C)} \operatorname{Arr}(C), \\ 0 & \text { otherwise. }\end{cases}
$$

It becomes an $A$-algebra in an evident way. There is another way, that will be useful, to define this algebra. A semigroup (respectively, monoid) with zero is a usual semigroup (respectively, monoid), say $S$, together with a two-sided absorbing element $0_{S}$, that is, for all $x \in S, x 0_{S}=0_{S}=0_{S} x$. A homomorphism between semigroups (respectively, monoid) with zero is a usual homomorphism of semigroups (monoids) 
that preserves the zeroes. The contracted algebra $A_{0}[S]$ (see [19]) is then defined as the free $A$-module with basis $S$ together with the associative product given for every $x, y \in S$ by

$$
x \times y= \begin{cases}x y & \text { if } x y \neq 0_{S} \\ 0 & \text { if } x y=0_{S}\end{cases}
$$

We observe that it is a unital algebra when $S$ is a monoid with zero. Now, the arrows of any semicategory (respectively, category) $C$ together with a new element $0_{C}$ added form a semigroup with zero with multiplication given for every $f, g \in \operatorname{Arr}(C) \sqcup\left\{0_{C}\right\}$ by $f g=f \circ g$ if, and only if, $(f, g) \in \operatorname{Arr}(C) \times_{0 \mathrm{~b}(C)} \operatorname{Arr}(C)$, and $f g=0_{C}$ otherwise. Moreover, any functor $\phi: C \rightarrow D$ between two semicategories (respectively, categories) defines a homomorphism of semigroups with zero $\phi^{0}: \operatorname{Arr}(C) \sqcup\left\{0_{C}\right\} \rightarrow \operatorname{Arr}(D) \sqcup\left\{0_{D}\right\}$ by extending $\phi$ with $\phi^{0}\left(0_{C}\right)=0_{D}$. This provides a functor from the category of all semi-categories (respectively, categories) to the category of semigroups with zero. Moreover, it is clear that $A\langle C\rangle \cong A_{0}[\operatorname{Arr}(C) \sqcup\{0\}]$ (as $A$ - and $R$ algebras). We observe that $\operatorname{Arr}(C) \sqcup\{0\}$ is a monoid with zero if, and only if, $C$ is a category with only one object, that is, $\operatorname{Arr}(C)$ is a usual monoid (under composition). In this case, $A\langle C\rangle$ is isomorphic to the usual (unital) algebra $A[\operatorname{Arr}(C)]$ of the monoid $\operatorname{Arr}(C)$. If $\mathrm{Ob}(C)$ is finite, then $A\langle C\rangle$ is a unital algebra with identity $\sum_{x \in \mathrm{Ob}(C)} \mathrm{id}_{x}$.

A finite decomposition semigroup (respectively, monoid) with zero $S$ is a semigroup (respectively, monoid) with zero such that for all $x \in S \backslash\left\{0_{S}\right\}$, the set $\left\{(y, z) \in S^{2}: x=y z\right\}$ is finite (it is a generalization of the property (D) from [18]). This makes it possible to consider a topological completion for the contracted algebra $A_{0}[S]$. As module $A_{0}[S]$ is isomorphic to the module $A^{\left(S \backslash\left\{0_{S}\right\}\right)}$ of all finitely supported maps from $S \backslash\left\{0_{S}\right\}$ to $A$. Under this isomorphism, we may equip $A_{0}[S]$ with the product topology, with $A$ (and $R$ ) discrete, inherited from the product $A$-module $A^{S\left\{\left\{0_{s}\right\}\right.}$. Its topological completion is $A^{S \backslash\left\{0_{S}\right\}}$ with its product topology. Any element $f$ of $A^{S \backslash\left\{0_{S}\right\}}$ may be represented in a unique way as a formal series $\sum_{x \in S \backslash\left\{0_{S}\right\}}\langle f \mid x\rangle x$ as in Section 2. Moreover, because $S$ is a finite decomposition semigroup with zero, the multiplication of $A_{0}[S]$ may be uniquely extended by uniform continuity to an associative and jointly continuous multiplication on $A^{S\left\{\left\{0_{S}\right\}\right.}$ given by

$$
\begin{gathered}
\left(\sum_{x \in S \backslash\left\{0_{S}\right\}}\langle f \mid x\rangle x\right)\left(\sum_{x \in S \backslash\left\{0_{S}\right\}}\langle g \mid x\rangle x\right) \\
=\sum_{x \in S \backslash\left\{0_{S}\right\}}\left(\sum_{y z=x}\langle f \mid y\rangle\langle g \mid z\rangle\right) x .
\end{gathered}
$$

The module $A^{S \backslash\left\{0_{S}\right\}}$ with this product is called the total contracted algebra of $S$, and it is denoted by $A_{0}[[S]]$. It satisfies the following universal problem. Let $S$ be a finite decomposition semigroup (respectively, monoid) with zero, let $B$ be a complete topological $R$-algebra (respectively, unital $R$-algebra), and let $\phi: A_{0}[S] \rightarrow B$ be a continuous homomorphism of algebras (if $S$ is a monoid and $B$ is unital, then we also assume that $\left.\phi\left(1_{S}\right)=1_{B}\right)$, then there exists a unique continuous homomorphism of algebras $\hat{\phi}$ : $A_{0}[[S]] \rightarrow B$ such that $\widehat{\phi}(f)=\phi(f)$ for every $f \in A_{0}[S]$ (in addition, if $S$ is a monoid, and $B$ is unital, then $\widehat{\phi}$ respects the units). All the details of this construction may be found in $[20]$.

In a similar way, let us call a finite decomposition semicategory (respectively, category) a semicategory (respectively, category) $C$ such that for every $f \in \operatorname{Arr}(C)$, the set $\{(g, h) \in$ $\left.\operatorname{Arr}(C) \times_{\mathrm{ob}(C)} \operatorname{Arr}(C): f=g \circ h\right\}$ is finite (it is again a generalization of the property (D) from [18] that may be found in [21-23]). Once more this allows us to define a topological completion for $A\langle C\rangle$ given as the $A$-algebra $A\langle\langle C\rangle\rangle$ (which is $A^{\operatorname{Arr}(C)}$ as an $A$-module) of all formal series $\sum_{f \in \operatorname{Arr}(C)}\langle S|$ $f\rangle f$, with product $\left(\sum_{f \in \operatorname{Arr}(C)}\langle S \mid f\rangle f\right) \times\left(\sum_{f \in \operatorname{Arr}(C)}\langle T|\right.$ $f\rangle f)=\sum_{f \in \operatorname{Arr}(C)}\left(\sum_{(g, h) \in \operatorname{Arr}(C) \times \times_{\mathrm{ob}(C)} \operatorname{Arr}(C) ; f=g \circ h}\langle S \mid g\rangle\langle T|\right.$ $h\rangle) f=\sum_{f \in \operatorname{Arr}(C)}\left(\sum_{g \times h=f}\langle S \mid g\rangle\langle T \mid h\rangle\right) f$.

Remark 4. Let $S_{1}, \ldots, S_{n} \in A\langle\langle C\rangle\rangle$. We have

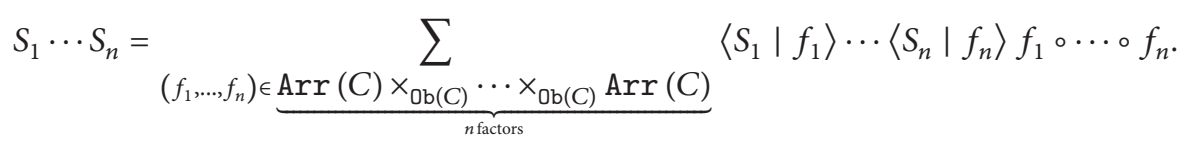

It is not difficult to see that $C$ is a finite decomposition semicategory (respectively, category) if, and only if, $\operatorname{Arr}(C) \sqcup$ $\left\{0_{C}\right\}$ is a finite decomposition semigroup, and in one of these equivalent cases, $A\langle\langle C\rangle\rangle \cong A_{0}\left[\left[\operatorname{Arr}(C) \sqcup\left\{0_{C}\right\}\right]\right]$ (as topological $A$ - and $R$-algebras). We observe that if $C$ is a category, then $\sum_{x \in \mathrm{Ob}(C)} \mathrm{id}_{x}$ is the two-sided identity of $A\langle\langle C\rangle\rangle$. The $R$-algebra $A\langle\langle C\rangle\rangle$ satisfies the following universal property: let $B$ be a complete topological $R$-algebra, and let $\phi$ be a continuous homomorphism of algebras from $A\langle C\rangle$ (with the product topology inherited from $A\langle\langle C\rangle\rangle$ ) to $B$. Then, there is a unique continuous homomorphism of
$R$-algebras $\widehat{\phi}: A\langle\langle C\rangle\rangle \rightarrow B$ such that $\widehat{\phi}(p)=\phi(p)$ for every $p \in A\langle C\rangle$. Let $C$ be a semicategory (respectively, a category). Let $\phi: \operatorname{Arr}(C) \rightarrow R\langle\langle C\rangle\rangle$ such that if $(f, g) \in$ $\operatorname{Arr}(C) \times_{\mathrm{ob}(C)} \operatorname{Arr}(C)$, then $\phi(f \circ g)=\phi(f) \phi(g)$, (and if $C$ is a category, $\phi\left(\mathrm{id}_{x}\right)=\mathrm{id}_{x}$ for every $\left.x \in \mathrm{Ob}(C)\right)$. If $C$ is a semicategory, then $\phi$ is a functor between $C$ and $A\langle\langle C\rangle\rangle$ (seen as a semicategory with only one object). If $C$ is a category, then this is no more a functor because it should be the case that $\phi\left(\mathrm{id}_{x}\right)=\sum_{x \in \mathrm{Ob}(C)} \mathrm{id}_{x}$ (the unit of $\left.A\langle\langle C\rangle\rangle\right)$. In any case, it is a homomorphism of semigroups with zero from $\operatorname{Arr}(C) \sqcup$ $\{0\}$ to $A\langle\langle C\rangle\rangle$. Therefore, it may be extended uniquely to 
a homomorphism of algebras $\phi: A\langle C\rangle \rightarrow A\langle\langle C\rangle\rangle$ by linearity. If it happens that it is continuous, then it may be itself extended as a continuous endomorphism of algebra $\phi: A\langle\langle C\rangle\rangle \rightarrow A\langle\langle C\rangle\rangle$. It satisfies $\phi\left(\sum_{f \in \operatorname{Arr}(C)}\langle S \mid f\rangle f\right)=$ $\sum_{f \in \operatorname{Arr}(C)}\langle S \mid f\rangle \phi(f)$. If $C$ is a category, then we observe that $\phi\left(\sum_{x \in \mathrm{Ob}(C)} \mathrm{id}_{x}\right)=\sum_{x} \phi\left(\mathrm{id}_{x}\right)=\sum_{x} \mathrm{id}_{x}$ so that $\phi$ respects the unit.

Let $G$ be a directed graph or quivers (see [24]), that is, it is given by a set $\mathscr{V}(G)$ of vertices, a set $\mathscr{E}(G)$ of edges, and two maps $d_{0}^{G}$ and $d_{1}^{G}$ (or simply $d_{0}, d_{1}$ when there is no risk of confusion) : $\mathscr{E}(G) \rightarrow \mathscr{V}(G)$. We may define the free semicategory $G^{+}$(respectively, category $G^{*}$ ) generated by $G$ as follows: $\mathrm{Ob}\left(G^{+}\right)=\mathrm{Ob}\left(G^{*}\right)=\mathscr{V}(G), \operatorname{Arr}\left(G^{+}\right)=$ $\left\{\left(e_{1}, \ldots, e_{n}\right): \mathscr{E}(V)^{n}: n \geq 1, d_{0}\left(e_{i+1}\right)=d_{1}\left(e_{i}\right), i=\right.$ $1, \ldots, n-1\}$ (in particular for $n=1,(e) \in \operatorname{Arr}\left(G^{+}\right)$ for all $e \in \mathscr{E}(G)$, and these $n$-tuples $\left(e_{1}, \ldots, e_{n}\right)$ are called paths of length $n), \operatorname{Arr}\left(G^{*}\right)=\{(\epsilon, x): x \in \mathscr{V}(G)\} \sqcup$ $\operatorname{Arr}\left(G^{+}\right)$(where $\epsilon$ denotes the empty word, and $(\epsilon, x)$ is a path of length zero), $\partial_{0}\left(e_{1}, \ldots, e_{n}\right)=d_{0}\left(e_{n}\right), \partial_{1}\left(e_{1}, \ldots, e_{n}\right)=$ $d_{1}\left(e_{1}\right), \partial_{0}(\epsilon, x)=x=\partial_{1}(\epsilon, x), \iota(\epsilon, x)=x$, for every $\left(e_{1}, \ldots, e_{m}\right),\left(f_{1}, \ldots, f_{n}\right) \in \operatorname{Arr}\left(G^{+}\right)$such that $d_{0}\left(e_{m}\right)=d_{1}\left(f_{1}\right)$, then $\left(e_{1}, \ldots, e_{m}\right) \circ\left(f_{1}, \ldots, f_{n}\right)=\left(e_{1}, \ldots, e_{m}, f_{1}, \ldots, f_{n}\right)$, $\left(e_{1}, \ldots, e_{m}\right) \circ\left(\epsilon, d_{0}\left(e_{m}\right)\right)=\left(e_{1}, \ldots, e_{m}\right)=\left(\epsilon, d_{1}\left(e_{1}\right)\right) \circ$ $\left(e_{1}, \ldots, e_{m}\right)$, and $(\epsilon, x) \circ(\epsilon, x)=(\epsilon, x)$ for every $x \in \mathscr{V}(G)$. It is easy to check that $G^{+}$and $G^{*}$ are indeed a semicategory and a category, respectively. Moreover, $G^{*}$ is obtained from $G^{+}$by free adjunctions of units $(\epsilon, x)$ for each object $x$ (and the obvious extensions of $\partial_{j}, j=0,1$, and of the composition). This means the following: let $C$ be a semicategory. We denote by $C^{1}$ the category obtained from $C$ by adjunctions of the identities on each object of $C$, the trivial extension of the domain and codomain maps, and the composition. Then, for every category $D$ seen as a semicategory in an evident way, and any functor $\phi: C \rightarrow D$ there is a unique functor $\phi^{1}: C^{1} \rightarrow D$ such that $\phi^{1}(f)=\phi(f)$ for every $f \in \operatorname{Arr}(C)$. A morphism of directed graph $\left(\phi_{V}, \phi_{E}\right)$ from $G_{1}$ to $G_{2}$ is a pair of maps $\phi_{V}: \mathscr{V}\left(G_{1}\right) \rightarrow \mathscr{V}\left(G_{2}\right), \phi_{E}: \mathscr{E}\left(G_{1}\right) \rightarrow \mathscr{E}\left(G_{2}\right)$ such that $\phi_{V} \circ d_{j}^{G_{1}}=d_{j}^{G_{2}} \circ \phi_{E}, j=0,1$. We observe that any semicategory (respectively, category) is obviously also a directed graph by forgetting some of its structure. Moreover, any functor between semi-categories (respectively, categories) is also a morphism between the underlying directed graphs. The following universal property is satisfied. Let $G$ be a directed graph, and let $C$ be a semicategory (respectively, category). Let $\left(\phi_{V}, \phi_{E}\right)$ be a morphism between $G$ and $C$ seen as a directed graph. Then, there is a unique functor $\bar{\phi}$ from $G^{+}$ (respectively, $G^{*}$ ) to $C$ such that for every $e \in \mathscr{E}(G), \bar{\phi}((e))=$ $\phi_{E}(e)$, and $\bar{\phi}(x)=\phi_{V}(x)$ for every $x \in \mathscr{V}(G)$.

Let $G$ be a directed graph. Let $S$ be a semigroup (respectively, monoid) with zero. Any morphism of directed graphs between $G$ to $S$, seen as a directed graph with only one vertex, is then given by a set-theoretic map $\phi_{E}: \mathscr{E}(G) \rightarrow S$ without any property to satisfy. Let $\phi_{E}: \mathscr{E}(G) \rightarrow A\left\langle\left\langle G^{*}\right\rangle\right\rangle$. This gives rise to a map $\phi: \operatorname{Arr}\left(G^{+}\right) \rightarrow A\left\langle\left\langle G^{*}\right\rangle\right\rangle$ (respectively, $\phi:$ $\left.\operatorname{Arr}\left(G^{*}\right) \rightarrow A\left\langle\left\langle G^{*}\right\rangle\right\rangle\right)$ by $\phi\left(e_{1}, \ldots, e_{n}\right)=\phi_{E}\left(e_{1}\right) \cdots \phi_{E}\left(e_{n}\right)$ (and in addition, $\phi\left(\mathrm{id}_{x}\right)=\mathrm{id}_{x}$ for each $x \in \mathscr{V}(G)$ ) which is shown to be a functor between semi-categories. It is unique with the property that $\widehat{\phi}((e))=\phi_{E}(e)$ for every $e \in \mathscr{E}(G)$ (and in addition, $\widehat{\phi}\left(\mathrm{id}_{x}\right)=\mathrm{id}_{x}$ for each object $\left.x\right)$.

It is quite clear that $G^{+}$(respectively, $G^{*}$ ) is a finite decomposition semicategory (respectively, category), or equivalently, $\operatorname{Arr}\left(G^{+}\right) \sqcup\{0\}$ (respectively, $\operatorname{Arr}\left(G^{*}\right) \sqcup\{0\}$ ) is a finite decomposition semigroup with zero (and even a finite decomposition monoid with zero if $G$ has only one vertex). Moreover $G^{+}$(respectively, $G^{*}$ ) satisfies a stronger property. It is a locally finite semicategory (respectively, category). Let $C$ be a semicategory (respectively, a category). Let $f \in \operatorname{Arr}(C)$. Any sequence $\left(f_{1}, \ldots, f_{n}\right)$ of composable arrows in $C$ (where in addition we assume that $f_{i}$ is not an identity for each $i=1, \ldots, n$, if $C$ is a category) is called a proper decomposition of length $n$ of $f$, if, and only if, $f=f_{1} \circ \cdots \circ f_{n}$. A proper decomposition of $f$ is then a proper decomposition of length $n$ of $f$ for some $n$. The set of all proper decompositions of length $n$ of $f$ is denoted by $D_{n}(f)$, and the set of all proper decompositions of $f$ is denoted by $D(f)=\bigcup_{n \geq 0} D_{n}(f)$. We assume that each identity arrow has only one decomposition, which has length zero, the empty decomposition (if $C$ is a category). We observe that for each $x \in \mathrm{Ob}(C)$ (if $C$ is a category), $D_{0}\left(\mathrm{id}_{x}\right)=\{\emptyset\}$, and $D_{n}\left(\mathrm{id}_{x}\right)=\emptyset$ for every $n>0$, while $D_{0}(f)=\emptyset, D_{1}(f)=\{f\}$ for every nonidentity arrow of $C$. A semicategory (respectively, category) is said to be locally finite [21-23] if for every arrow $f, D(f)$ is a finite set. A locally finite semicategory (respectively, category) $C$ is also a finite decomposition semicategory (respectively, category) because $D_{2}(f)$ is assumed to be finite (and this suffices for the case of semicategories because $D_{2}(f)=\left\{(g, h) \in \operatorname{Arr}(C) \times \times_{0 \mathrm{~b}(C)} \operatorname{Arr}(C)\right.$ : $f=g \circ h\})$, and $\left\{(g, h) \in \operatorname{Arr}(C) \times \times_{\mathrm{ob}(C)} \operatorname{Arr}(C): f=\right.$ $g \circ h\}=D_{2}(f) \cup\left\{\left(\operatorname{id}_{\partial_{1}(f)}, f\right),\left(f, \operatorname{id}_{\partial_{0}(f)}\right)\right\}$ (if $C$ is a category). In particular, in a locally finite category, no nonidentity arrow may be left or right invertible. Indeed, if $f$ is right invertible, then there is $g$ such that $f \circ g=\operatorname{id}_{\partial_{0}(g)}$ (so that $\left.\partial_{1}(f)=\partial_{0}(g)\right)$, and then $(f, g, f, g, f, g, \ldots, f, g, f)$ is a proper decomposition of $f$ of arbitrary length.

Equivalently, a semigroup (respectively, monoid) with zero $S$ is said to be locally finite [20] if for every $x \neq 0_{S}$, for all $n$, the set of all $n$-tuples $\left(x_{1}, \ldots, x_{n}\right),\left(x_{i} \neq 1_{S}, i=1, \ldots, n\right.$, when $S$ is assumed to be a monoid), such that $x_{1} \cdots x_{n}=x$ is finite. It is clear that a semicategory (respectively, category) is locally finite, if, and only if, the semigroup (or monoid if $C$ has only one object since in this case $\operatorname{Arr}(C)$ is actually a monoid) with zero $\operatorname{Arr}(C) \sqcup\{0\}$ is locally finite.

For a locally finite semicategory (respectively, category), we define the length $\ell(f)$ of an arrow $f$ as the supremum in $\mathbb{N}$ of the lengths of decompositions of $f$. In particular, if $C$ is a locally finite category, $\ell(f)=0$ if, and only if, $f$ is an identity. For each free semicategory $G^{+}$(respectively, category $\left.G^{*}\right)$ over a directed graph $G$, the length of a path $\left(e_{1}, \ldots, e_{n}\right) \epsilon$ $\operatorname{Arr}\left(G^{+}\right)$is $n$, while the length of an identity $(\epsilon, x) \in \operatorname{Arr}\left(G^{*}\right)$ is zero.

Let $C$ be a locally finite semicategory (respectively, category). For every $\ell>0$ (respectively, $\ell \geq 0$ ), let us define $\operatorname{Arr}(C)_{\ell}=\{f \in \operatorname{Arr}(C): \ell(f)=\ell\}$. In particular, in a locally finite category, $\operatorname{Arr}(C)_{0}=\left\{\mathrm{id}_{x}: x \in \mathrm{Ob}(C)\right\}$. 
It is clear that in a locally finite semicategory (respectively, category), $\operatorname{Arr}(C)=\bigcup_{\ell>0} \operatorname{Arr}(C)_{\ell}$ (respectively, $\operatorname{Arr}(C)=$ $\left.\bigcup_{\ell \geq 0} \operatorname{Arr}(C)_{\ell}\right)$ which is a disjoint sum. According to [25], we have $\ell(f \circ g) \geq \ell(f)+\ell(g)$ for every $(f, g) \in$ $\operatorname{Arr}(C) \times \times_{\mathrm{Ob}(C)} \operatorname{Arr}(C)$. This length is extended to an order function $\omega: A\langle\langle C\rangle\rangle \rightarrow \mathbb{N} \cup\{\infty\}$ defined by $\omega(S)=\inf \{\ell(f):$ $\langle S \mid f\rangle \neq 0\}$ (the infimum being taken in $\mathbb{N} \cup\{\infty\}$ ). In particular, $\omega(S)=+\infty$ if, and only if, $S=0$. The following hold.

(1) $\omega\left(\mathrm{id}_{x}\right)=0$ for every object $x$ (if $C$ is a category).

(2) $\omega(S+T) \geq \min \{\omega(S), \omega(T)\}$.

(3) $\omega(S T) \geq \omega(S) \omega(T)$.

It is clear that the algebra $A\langle\langle C\rangle\rangle$ of a locally finite semicategory (respectively, category) $C$ is filtered by this order function. Another way to define this filtration is the following. Let us define $\mathfrak{M}_{\geq n}(A)=\{S \in A\langle\langle C\rangle\rangle \mid \omega(S) \geq n\}$ for each $n \geq 0$. Therefore, $\mathfrak{M}_{\geq 0}(A)=A\langle\langle C\rangle\rangle$, and $\mathfrak{M}_{\geq 1}(A)=$ $\left\{S \in A\langle\langle C\rangle\rangle:\left\langle S \mid \mathrm{id}_{x}\right\rangle=0, x \in \mathrm{Ob}(C)\right\}$ is a twosided ideal in $A\langle\langle C\rangle\rangle$ (improper if $C$ is a semicategory). It is clear that $\bigcap_{n \geq 0} \mathfrak{M}_{\geq n}(A)=(0)$ so that the filtration is separated and decreasing. With this filtration, $A\langle\langle C\rangle\rangle$ becomes a (Hausdorff) complete topological $A$-algebra (with $A$ and $R$ discrete) when $\left(\mathfrak{M}_{\geq n}(A)\right)_{n}$ is considered as a basis of neighborhoods of zero (see $[20,26])$. Observe that if there is some $\ell$ such that $\operatorname{Arr}(C)_{\ell}$ is infinite, then this topology is strictly finer than the product topology (in any case, namely, $\operatorname{Arr}(C)_{\ell}$ is either finite or infinite for each $\ell$, then it is finer since the projections $S \mapsto\langle S \mid f\rangle$ are continuous in the topology induced by the filtration for all $f$ ). For instance, let $\left(f_{n}\right)_{n \geq 0}$ be an infinite sequence of arrows such that $f_{m} \neq f_{n}$ for each $m \neq n$, and $\ell\left(f_{n}\right)=\ell$ for each $n$. Let $S_{n}=\sum_{k=0}^{n} f_{k}$. Then, $\left(S_{n}\right)_{n}$ converges to $S=\sum_{n \geq 0} f_{n}$ in $A\langle\langle C\rangle\rangle$ with the product topology, but does not converge for the topology induced by the filtration (since $\omega\left(S-S_{n}\right)=\ell$ for all $n$ ). Nevertheless when for each $\ell, \operatorname{Arr}(C)_{\ell}$ is finite, then both topologies coincide.

\section{Substitution of Series without Constant Terms}

Let $R$ be a commutative ring with a unit, and let $A$ be a commutative $R$-algebra with a unit. Let $C$ be any semicategory. Forgetting the composition of arrows, we obtain a directed graph $\underline{C}$. Let $\underline{C}^{+}$(respectively, $\underline{C}^{*}$ ) be the free semicategory (respectively, category) generated by $\underline{C}$. By universality of $\underline{C}^{+}$, there exists a unique functor $\pi: \underline{C}^{+} \rightarrow C$ such that $\pi((f))=$ $f$ for every $f \in \operatorname{Arr}(C)=\mathscr{E}(\underline{C})$. This functor is obviously onto on arrows (and the identity on objects). The paths in $\operatorname{Arr}\left(\underline{C}^{+}\right)$are denoted by $\vec{f}$, and for each such path there is a unique integer $\ell(\vec{f}) \geq 1$ and a unique sequence $\left(f_{1}, \ldots, f_{\ell(\vec{f})}\right)$ of composable arrows in $C$ such that $\vec{f}=\left(f_{1}, \ldots, f_{\ell(\vec{f})}\right)$. The composition of paths will be denoted by juxtaposition (we reserve $\circ$ for the composition in $C$ ). Let $f$ and $g$ be two composable arrows in $C$, and $\vec{f}, \vec{g} \in \operatorname{Arr}\left(\underline{C}^{+}\right)$such that $\pi(\vec{f})=f, \pi(\vec{g})=g$. Then, $\vec{f}$ and $\vec{g}$ are composable in $\underline{C}^{+}$. Indeed, $\partial_{0}(\vec{f})=\pi\left(\partial_{0}(\vec{f})\right)=\partial_{0}(\pi(\vec{f}))=\partial_{0}(f)=\partial_{1}(g)=$ $\partial_{1}(\pi(\vec{g}))=\pi\left(\partial_{1}(\vec{g})\right)=\partial_{1}(\vec{g})$. Because $\pi$ is a functor, we have then $\pi(\vec{f} \vec{g})=f \circ g=\pi(\vec{f}) \circ \pi(\vec{g})$. More generally, if $\left(f^{(1)}, \ldots, f^{(n)}\right)$ are composable in $C$ and for each $i, \vec{f}^{(i)} \in$ $\operatorname{Arr}\left(\underline{C}^{+}\right)$such that $\pi\left(\vec{f}^{(i)}\right)=f^{(i)}$, then $\left(\vec{f}^{(1)}, \ldots, \vec{f}^{(n)}\right)$ is an $n$-tuple of composable paths in $\underline{C}^{+}$and $\pi\left(\vec{f}^{(1)}, \ldots, \vec{f}^{(n)}\right)=$ $f_{1} \circ \cdots \circ f_{n}=\pi\left(\vec{f}^{(1)}\right) \circ \cdots \circ \pi\left(\vec{f}^{(n)}\right)$. We also observe that for every $\ell>0, \operatorname{Arr}\left(\underline{C}^{*}\right)_{\ell}=\operatorname{Arr}\left(\underline{C}^{+}\right)_{\ell}$.

Let $S \in A\left\langle\left\langle\underline{C}^{*}\right\rangle\right\rangle$ and $f \in \operatorname{Arr}(C)$. We define $S_{f}=$ $\sum_{\vec{f} \in \operatorname{Arr}\left(\underline{C}^{+}\right), \pi(\vec{f})=f}\langle S \mid \vec{f}\rangle \vec{f} \in R\left\langle\left\langle\underline{C}^{+}\right\rangle\right\rangle \subseteq R\left\langle\left\langle\underline{C}^{*}\right\rangle\right\rangle$. Let $\rho_{S}:$ $\underline{C} \rightarrow A\left\langle\left\langle\underline{C}^{*}\right\rangle\right\rangle$ be defined as the morphism of directed graphs $\rho_{S}(f)=S_{f}$ (when $A\left\langle\left\langle\underline{C}^{*}\right\rangle\right\rangle$ is a directed graph with only one vertex). Then, it is extended uniquely to a functor $\rho_{S}: \underline{C}^{*} \rightarrow A\left\langle\left\langle\underline{C}^{*}\right\rangle\right\rangle$ by $\rho_{S}(\vec{f})=\rho_{S}\left(f_{1}\right) \cdots \rho_{S}\left(f_{n}\right)$, and $\rho_{S}(\epsilon, x)=(\epsilon, x)$ for every object $x$ of $C$ (again, $A\left\langle\left\langle\underline{C}^{*}\right\rangle\right\rangle$ is seen as a semicategory with only one object). According to the universal property of $A\left\langle\underline{C}^{*}\right\rangle, \rho_{S}$ is uniquely extended by linearity to a homomorphism of algebras, again denoted $\rho_{S}$, from $A\left\langle\underline{C}^{*}\right\rangle$ to $A\left\langle\left\langle\underline{C}^{*}\right\rangle\right\rangle$ (we observe that in general it does not preserve the units since $A\left\langle\underline{C}^{*}\right\rangle$ is unital only when $\mathrm{Ob}(C)$ is finite; however $\rho_{S}\left(\mathrm{id}_{x}\right)=\mathrm{id}_{x}$ for each object $\left.x\right)$. We have $\rho_{S}(p)=\rho_{S}\left(\sum_{x \in \mathrm{Ob}(C)}\left\langle p \mid \operatorname{id}_{x}\right\rangle \mathrm{id}_{x}+\sum_{\vec{f}}\langle p \mid \vec{f}\rangle \vec{f}\right)=$ $\sum_{x \in \mathrm{Ob}(C}\left\langle p \mid \operatorname{id}_{x}\right\rangle \rho_{S}\left(\mathrm{id}_{x}\right)+\sum_{\vec{f}}\langle p \mid \vec{f}\rangle \rho_{S}(\vec{f})=\sum_{x}\langle p|$ $\left.\operatorname{id}_{x}\right\rangle \operatorname{id}_{x}+\sum_{\vec{f}}\langle p \mid \vec{f}\rangle S_{f_{1}} \cdots S_{f_{\ell(\vec{f})}}$ for each $p \in A\left\langle\underline{C}^{*}\right\rangle$.

Lemma 5. The homomorphism $\rho_{S}$ is continuous (for the filtrations).

Proof. By linearity it is sufficient to prove that $\vec{g} \mapsto \rho_{S}(\vec{g})$ is continuous. We have $\omega\left(\rho_{S}(\vec{g})\right)=\omega\left(S_{g_{1}} \cdots S_{g_{\ell(\vec{g})}}\right) \geq$ $\sum_{i=1}^{\ell(\vec{g})} \omega\left(S_{g_{i}}\right)$. Now, $S_{g_{i}}=\sum_{\vec{h}^{(i)}, \pi\left(\vec{h}^{(i)}\right)=g_{i}}\left\langle S \mid \vec{h}^{(i)}\right\rangle \vec{h}^{(i)}, i=$ $1, \ldots, \ell(\vec{g})$. Therefore, $\omega\left(S_{g_{i}}\right) \geq \omega(S)$ for all $i=1, \ldots, \ell(\vec{g})$, and then $\omega\left(\rho_{S}(\vec{g})\right) \geq \ell(g) \omega(S)$ so that $\rho_{S}$ is continuous for the filtrations.

From now on let us assume that $C$ is finite, that is, the sets $\operatorname{Arr}(C)=\mathscr{E}(\underline{C})$ and $\mathrm{Ob}(C)=\mathscr{V}(\underline{C})$ are finite (if $C$ is a category, then it is sufficient to assume that $\operatorname{Arr}(C)$ is finite, because $\partial_{j}$ is onto, $j=0,1$, but when $C$ is a semicategory that lacks identities, then we need in addition to assume that $\mathrm{Ob}(C)$ is finite, because $\partial_{j}, j=0,1$, is not assumed to be onto anymore). Then, for each $\ell \in \mathbb{N}$, there are only finitely many elements in $\operatorname{Arr}\left(\underline{C}^{*}\right)_{\ell}$ (in particular, $\operatorname{Arr}\left(\underline{C}^{*}\right)_{0}=\left\{\operatorname{id}_{x}\right.$ : $x \in \mathrm{Ob}(C)\})$, so that the product topology and the filtration coincide. According to Lemma 5, $\rho_{S}$ is continuous for the product topologies, and we may extend it by continuity to the completion $A\langle\langle C\rangle\rangle$ of $A\langle C\rangle$ for the product topologies. We obtain a continuous algebra endomorphism, again denoted by $\rho_{S}$, of $A\langle\langle C\rangle\rangle$ such that $\rho_{S}(T)=\rho_{S}\left(\sum_{x \in \mathrm{Ob}(C)}\left\langle T \mid \mathrm{id}_{x}\right\rangle \mathrm{id}_{x}+\right.$ $\left.\sum_{\vec{f}}\langle T \mid \vec{f}\rangle \vec{f}\right)=\sum_{x \in \mathrm{Ob}(C)}\left\langle T \mid \mathrm{id}_{x}\right\rangle \mathrm{id}_{x}+\sum_{\vec{f}}\langle T \mid \vec{f}\rangle \rho_{S}(\vec{f})$. In particular, $\rho_{S}\left(\sum_{x \in \mathrm{Ob}(C)} \mathrm{id}_{x}\right)=\sum_{x \in \mathrm{Ob}(C)} \rho_{S}\left(\mathrm{id}_{x}\right)=\sum_{x \in \mathrm{Ob}(C)} \mathrm{id}_{x}$, so that both $\rho_{S}: A\langle C\rangle \rightarrow A\langle\langle C\rangle\rangle$ and its continuous extension $\rho_{S}: A\langle\langle C\rangle\rangle \rightarrow A\langle\langle C\rangle\rangle$ respect the units $\left(\sum_{x \in \mathrm{Ob}(C)} \mathrm{id}_{x} \epsilon\right.$ $A\langle C\rangle$ because $\mathrm{Ob}(C)$ is finite). 
Lemma 6. The series $\rho_{S}(T)$ is given by the following.

(1) $\left\langle\rho_{S}(T) \mid \mathrm{id}_{x}\right\rangle=\left\langle T \mid \mathrm{id}_{x}\right\rangle$ for every $x \in \mathrm{Ob}(C)$.

$(2)\left\langle\rho_{S}(T) \quad \mid \quad \vec{f}\right\rangle=\sum_{\ell=1}^{\ell(\vec{f})} \sum_{\left(\vec{f}^{(1)}, \ldots, \vec{f}^{(\ell)}\right) \in D_{k}(\vec{f})}\langle T$ $\left.\left(\pi\left(\vec{f}^{(1)}\right), \ldots, \pi\left(\vec{f}^{(\ell)}\right)\right)\right\rangle\left\langle S \mid \vec{f}^{(1)}\right\rangle \cdots\left\langle S \mid \vec{f}^{(\ell)}\right\rangle$ for every $\vec{f} \in \operatorname{Arr}\left(\underline{C}^{+}\right)$.

Proof. We have

$$
\begin{aligned}
\rho_{S}(T)= & \sum_{x \in \operatorname{Ob}(C)}\left\langle T \mid \operatorname{id}_{x}\right\rangle \mathrm{id}_{x}+\sum_{\ell \geq 1} \sum_{\vec{f} \in \operatorname{Arr}\left(\underline{C}^{*}\right)_{\ell}}\langle T \mid \vec{f}\rangle \\
& \times\left(\sum_{\substack{\vec{f}^{(1)} \in \operatorname{Arr}\left(\underline{C}^{+}\right) \\
\pi\left(\vec{f}^{(1)}\right)=f_{1}}}\left\langle S \mid \vec{f}^{(1)}\right\rangle \vec{f}^{(1)}\right) \\
& \ldots\left(\begin{array}{c}
\sum_{\substack{\vec{f}^{(\ell)} \in \operatorname{Arr}\left(\underline{C}^{+}\right) \\
\pi\left(\vec{f}^{(\ell)}\right)=f_{\ell}}}\left\langle S \mid \vec{f}^{(\ell)}\right\rangle \vec{f}^{(\ell)}
\end{array}\right) .
\end{aligned}
$$

(1) Let $x \in \mathrm{Ob}(C)$. We have $\left\langle\rho_{S}(T) \mid \mathrm{id}_{x}\right\rangle=\left\langle T \mid \mathrm{id}_{x}\right\rangle+$ $\sum_{\vec{f}}\langle T \mid \vec{f}\rangle\left\langle\rho_{S}(\vec{f}) \mid \operatorname{id}_{x}\right\rangle$. We have $\left\langle\rho_{S}(\vec{f}) \mid \operatorname{id}_{x}\right\rangle=$ $\left\langle\rho_{S}\left(f_{1}\right) \cdots \rho_{S}\left(f_{\ell(\vec{f})}\right) \mid \operatorname{id}_{x}\right\rangle=\sum_{\vec{h}_{1}, \ldots, \vec{h}_{\ell(\vec{f})}, \pi\left(\vec{h}_{i}\right)=f_{i}}\langle S|$ $\left.\vec{h}_{1}\right\rangle \cdots\left\langle S \mid \vec{h}_{\ell(\vec{f})}\right\rangle\left\langle\vec{h}_{1} \cdots \vec{h}_{\ell(\vec{f})} \mid \operatorname{id}_{x}\right\rangle=0$.

(2) Let $\vec{f} \in \operatorname{Arr}\left(\underline{C}^{+}\right)$(that is, $\ell(\vec{f})>0$ ). Each proper decomposition of $\vec{f}$ of the form $\vec{f}=\vec{f}^{(1)} \cdots \vec{f}^{(\ell)}$, $1 \leq \ell \leq \ell(\vec{f})$, occurs in the sum (6) with coefficient given by

$$
\left\langle T \mid\left(f_{1}, \ldots, f_{\ell}\right)\right\rangle\left\langle S \mid \vec{f}^{(1)}\right\rangle \cdots\left\langle S \mid \vec{f}^{(\ell)}\right\rangle,
$$

where $\pi\left(\vec{f}^{(i)}\right)=f_{i}$. Collecting all these coefficients yields the expected result for the coefficient of $\vec{f}$ in $\rho_{S}(T)$.

We observe that $\rho_{S}$ maps $A\left\langle\left\langle\underline{C}^{+}\right\rangle\right\rangle$to itself according to the first point of Lemma 6 . Let $I=\sum_{f \in \operatorname{Arr}(C)}(f)$. For every $f \in \operatorname{Arr}(C)=\mathscr{E}(\underline{C}), \rho_{I}((f))=(f)$, then for each $\vec{f}=$ $\left(f_{1}, \ldots, f_{\ell(\vec{f})}\right), \rho_{I}(\vec{f})=\vec{f}$. Therefore, $\rho_{I}(p)=p$ for every $p \in$ $A\left\langle\underline{C}^{*}\right\rangle$ (since we also have $\left.\rho_{I}\left(\mathrm{id}_{x}\right)=\mathrm{id}_{x}\right)$. Therefore, $\rho_{I}$ is the identity on $A\left\langle\left\langle\underline{C}^{*}\right\rangle\right\rangle$ (since $A\left\langle\underline{C}^{*}\right\rangle$ is dense in $A\left\langle\left\langle\underline{C}^{*}\right\rangle\right\rangle$, and $\rho_{I}$ is continuous). Moreover, we have $\operatorname{Arr}\left(\underline{C}^{*}\right)=\operatorname{Arr}\left(\underline{C}^{+}\right) \sqcup$ $\left\{\mathrm{id}_{x}: x \in \mathrm{Ob}(C)\right\}=\bigsqcup_{f \in \operatorname{Arr}(C)} \pi^{-1}(\{f\}) \sqcup\left\{\mathrm{id}_{x}: x \in \mathrm{Ob}(C)\right\}$. For every $f \in \operatorname{Arr}(C)$, then $\rho_{S}(f)=\sum_{\vec{f} \in \pi^{-1}(\{f\})}\langle S \mid \vec{f}\rangle \vec{f}$, so that $\rho_{S}(I)=\sum_{f \in \operatorname{Arr}(C)} \sum_{\vec{f} \in \pi^{-1}(\{f\})}\langle S \mid \vec{f}\rangle \vec{f}$. But the family $(\langle S \mid \vec{f}\rangle)_{\vec{f}}$ is summable, and by associativity of summable families, $\sum_{\vec{f}}\langle S \mid \vec{f}\rangle \vec{f}=\sum_{f \in \operatorname{Arr}(C)} \sum_{\vec{f} \in \pi^{-1}(\{f\})}\langle S \mid \vec{f}\rangle \vec{f}$. Finally for similar reasons, $S=\sum_{F \in \operatorname{Arr}\left(\underline{C}^{*}\right)}\langle S \mid F\rangle F=\sum_{x \in \mathrm{Ob}(C)}\langle S|$ $\left.\operatorname{id}_{x}\right\rangle \mathrm{id}_{x}+\sum_{\vec{f}}\langle S \mid \vec{f}\rangle \vec{f}=\sum_{x \in \mathrm{Ob}(C)}\left\langle S \mid \mathrm{id}_{x}\right\rangle \mathrm{id}_{x}+\rho_{S}(I)$, so that $\rho_{S}(I)=S-\sum_{x \in \mathrm{Ob}(C)}\left\langle S \mid \mathrm{id}_{x}\right\rangle \mathrm{id}_{x}$. This means that $I$ acts as a two-sided identity for the operation $(S, T) \in A\left\langle\left\langle\underline{C}^{+}\right\rangle\right\rangle^{2} \mapsto$ $\rho_{S}(T) \in A\left\langle\left\langle\underline{C}^{+}\right\rangle\right\rangle$.

Example 7. (1) Let $C$ be the semicategory with only one object and one arrow $x$. Then, $\underline{C}^{+} \cong \mathbb{N} \backslash\{0\}$, and $\underline{C}^{*} \cong \mathbb{N}$. Moreover, $R\left\langle\left\langle\underline{C}^{*}\right\rangle\right\rangle \cong R[[x]]$. Finally, $\rho_{S}(T)=T \circ(S-\langle S \mid 1\rangle 1)$, where $\circ$ denotes the usual substitution of formal power series (see for instance [27]), and $1=x^{0}$ is the unit of $R[[x]]$.

(2) Let $S$ be a finite semigroup. Then, $\underline{S}^{+} \cong S^{+}$(the free semigroup on $S$ ), and $\underline{S}^{*} \cong S^{*}$ (the free monoid on $S$, with identity the empty word $\epsilon)$. Therefore, $R\left\langle\left\langle\underline{S}^{*}\right\rangle\right\rangle \cong$ $R\left[\left[S^{*}\right]\right]$ (the large algebra of $S^{*}$ ). We have $\rho_{S}(T)=\langle T|$ $\epsilon\rangle \epsilon+\sum_{n \geq 1} \sum_{x_{1} \cdots x_{n} \in S^{+}, x_{i} \in S}\left\langle T \quad \mid \quad x_{1} \cdots x_{n}\right\rangle\left(\sum_{\pi\left(\vec{w}^{(1)}\right)=x_{1}}\langle S \quad\right.$ | $\left.\left.\vec{w}^{(1)}\right\rangle \vec{w}^{(1)}\right) \cdots\left(\sum_{\pi\left(\vec{w}^{(n)}\right)=x_{n}}\left\langle S \mid \vec{w}^{(n)}\right\rangle \vec{w}^{(n)}\right)$.

(3) Let $(P, \leq)$ be a finite poset. We define a semicategory $C(P,<)$, where $<$ is the strict partial order associated to $\leq$. Its set of objects is $P$. We have an arrow between $x$ and $y$ if, and only if, $y<x$. The composition is the obvious one. Now, $C(P,<)^{*}$ consists of the identities $x$ for every $x \in P$, and all the chains $x_{1}<\cdots<x_{n}$. Because $P$ is finite, it is clear that $\operatorname{Arr}\left(C(P,<)^{*}\right)$ is finite. Moreover $\pi\left(x_{1}<\cdots<x_{n}\right)=x_{1}<x_{n}$. We have $\rho_{S}(x<y)=\sum_{x<\cdots<y}\langle S \mid x<\cdots<y\rangle x<\cdots<y$. In particular, if $y$ covers $x$, then $\rho_{S}(x<y)=\langle S \mid x<y\rangle x<$ $y$. We have $\rho_{S}(T)=\sum_{x \in P}\langle T \mid x\rangle x+\sum_{n \geq 1} \sum_{x_{1}<\cdots<x_{n}}\langle T|$ $\left.x_{1}<\cdots<x_{n}\right\rangle\left(\sum_{x_{1}<\cdots<x_{2}}\left\langle S \mid x_{1}<\cdots<x_{2}\right\rangle x_{1}<\cdots<\right.$ $\left.x_{2}\right) \cdots\left(\sum_{x_{n-1}<\cdots<x_{n}}\left\langle S \mid x_{n-1}<\cdots<x_{n}\right\rangle x_{n-1}<\cdots<x_{n}\right)$.

We now denote $\rho_{S}(T)$ by $T \circ S$, and call it the substitution. We also define $\mathfrak{M}(A)=\left\{S \in \mathfrak{M}_{\geq 1}(A): \forall f \in \operatorname{Arr}(C),\langle S|\right.$ $(f)\rangle \neq 0\}$.

Lemma 8. (1) The set $A\left\langle\left\langle\underline{C}^{+}\right\rangle\right\rangle$is a monoid under $\circ$.

(2) Let $S \in A\left\langle\left\langle\underline{C}^{+}\right\rangle\right\rangle$be left (respectively, right) invertible (with respect to o). Then, for every $f \in \mathscr{A},\langle S \mid(f)\rangle$ is left (respectively, right) invertible in $A$, and so it is invertible since $A$ is commutative. In particular, $S \in \mathfrak{M}(A)$.

(3) Let $S \in A\left\langle\left\langle\underline{C}^{+}\right\rangle\right\rangle$such that for every $f \in \mathscr{C},\langle S \mid(f)\rangle$ is invertible in $A$ (in particular, $S \in \mathfrak{M}(A)$ ). Then, $S$ is invertible (with respect to $\circ$ ), its inverse $T$, denoted by $S^{\circ-1}$, is an element of $\mathfrak{M}(A)$ such that for every $f \in \operatorname{Arr}(C),\langle T \mid(f)\rangle$ is the inverse of $\langle S \mid(f)\rangle$. In particular, if $\langle S \mid(f)\rangle=1_{A}$ for every $f \in \operatorname{Arr}(C)$, then $S$ is invertible, and its inverse $T$ satisfies $\langle T|$ $(f)\rangle=1_{A}$ for every $f \in \operatorname{Arr}(C)$.

(4) If $A$ is a field say $\mathbb{K}$, then $\mathfrak{M}(A)$ is a group under $\circ$.

Proof. (1) It is already known that $\circ$ is a binary operation for $A\left\langle\left\langle\underline{C}^{+}\right\rangle\right\rangle$, with unit given by $I$. It remains to prove associativity. Actually we can prove the following. Let $S, T, U \in$ $R\left\langle\left\langle\underline{C}^{*}\right\rangle\right\rangle$. For every $x \in \mathrm{Ob}(C),\left\langle(S \circ T) \circ U \mid \mathrm{id}_{x}\right\rangle=$ $\left\langle S \circ T \mid \operatorname{id}_{x}\right\rangle=\left\langle S \mid \operatorname{id}_{x}\right\rangle=\left\langle S \circ(T \circ U) \mid \operatorname{id}_{x}\right\rangle$. Now, let $\vec{f}$ with $\ell(\vec{f})>0$. The computation of $S \circ(T \circ U)$ at $\vec{f}$ involves all proper decompositions $\vec{f}=\vec{f}^{(1)} \cdots \vec{f}^{(k)}$ into nontrivial paths, and then all the proper decompositions of each factor $\vec{f}^{(1)}=\vec{f}^{(1,1)} \cdots \vec{f}^{\left(1, \ell_{1}\right)}, \ldots, \vec{f}^{(k)}=\vec{f}^{(k, 1)} \cdots \vec{f}^{\left(k, \ell_{k}\right)}$. 
It is equivalent to sum first over all the proper decompositions $\vec{f}=\vec{f}^{(1,1)} \ldots \vec{f}^{\left(k, \ell_{k}\right)}$ and then to consider all manners of regrouping adjacent factors $\left(\vec{f}^{(1,1)} \cdots \vec{f}^{\left(1, \ell_{1}\right)}\right) \cdots\left(\vec{f}^{(k, 1)} \cdots\right.$ $\left.\vec{f}^{\left(k, \ell_{k}\right)}\right)$, which yields to the value of $(S \circ T) \circ U$ at $\vec{f}$.

(2) Let $S \in R\left\langle\left\langle\underline{C}^{+}\right\rangle\right\rangle$be left (respectively, right) invertible. Therefore there exists some $T \in R\left\langle\left\langle\underline{C}^{+}\right\rangle\right\rangle$such that $T \circ S=I$ (respectively, $S \circ T=I$ ). In particular, for every $f \in \operatorname{Arr}(C)$, $\langle T \mid(f)\rangle\langle S \mid(f)\rangle=\langle T \circ S \mid(f)\rangle=1$ (respectively, $\langle S|$ $(f)\rangle\langle T \mid(f)\rangle=\langle S \circ T \mid(f)\rangle=1)$, according to Lemma 6 .

(3) Let $S \in R\left\langle\left\langle\underline{C}^{+}\right\rangle\right\rangle$such that for every $f \in \operatorname{Arr}(C)$, $\langle S \mid(f)\rangle$ is invertible in $A$. For every $x \in \mathrm{Ob}(C)$, let us define $T_{(x)}=0$. For every $f \in \operatorname{Arr}(C)$, let us define $T_{(f)}=\langle S \mid(f)\rangle^{-1}$. Now, suppose that $\vec{f} \in \operatorname{Arr}\left(\underline{C}^{+}\right)$such that $\ell(\vec{f})>1$. By induction on $\ell(\vec{f})$, we define $T_{(\vec{f})}=$ $-\langle S \quad \mid \quad(\pi(\vec{f}))\rangle^{-1} \sum_{k=2}^{\ell(\vec{f})} \sum_{\left(\vec{f}^{(1)}, \ldots, \vec{f}^{(k)}\right) \in D_{k}(\vec{f})}\langle S \quad| \quad \pi\left(\vec{f}^{(1)}\right) \cdots$ $\left.\pi\left(\vec{f}^{(k)}\right)\right\rangle T_{\left(\vec{f}^{(1)}\right)} \cdots T_{\left(\vec{f}^{(k)}\right)}$. We define $T=\sum_{\vec{f} \in \operatorname{Arr}\left(\underline{C}^{*}\right)} T_{(\vec{f})} \vec{f}$. It is clear that $T \in \mathfrak{M}(A)$, and that $\langle T \mid(f)\rangle=\langle S \mid(f)\rangle^{-1}$ for every $f \in \operatorname{Arr}(C)$. It is easy to see that $S \circ T=I$ (because $T$ is built to satisfy this property), so that $S$ is left invertible. Since for every $f \in \operatorname{Arr}(C),\langle T \mid(f)\rangle=\langle S \mid(f)\rangle^{-1}$ is invertible in $A$, it follows that $T$ also is left invertible. Therefore there is $S^{\prime}$ such that $T \circ S^{\prime}=I$, then $S=S \circ I=S \circ T \circ S^{\prime}=S^{\prime}$ so that $S$ is invertible with (two-sided) inverse $T$. The second fact becomes obvious.

(4) It is clear that $\mathfrak{M}(A)$ is a submonoid of $\left(A\left\langle\left\langle\underline{C}^{+}\right\rangle\right\rangle, \circ, I\right)$ (since $I \in \mathfrak{M}(A)$, and for every $\vec{f}$ such that $\ell(\vec{f})=1,\langle S \circ T|$ $\vec{f}\rangle=\langle S \mid \vec{f}\rangle\langle T \mid \vec{f}\rangle \neq 0$ whenever $S, T \in \mathfrak{M}(A))$. To prove that $\mathfrak{M}(A)$ is a group under $\circ$ (if $A$ is the field $\mathbb{K}$ ), it suffices to prove that any element of $\mathfrak{M}(A)$ is invertible (with respect to o) which is clear according to the previous point.

\section{Coordinate Hopf Algebras for Groups of Invertible Series with respect to Multiplication and Substitution}

In this section we define two $R$-groups of series over a category, and we use the result from Section 3 to introduce their coordinate Hopf algebras.

6.1. Group of (Unipotent) Invertible Series. Let $A$ be a commutative $R$-algebra with a unit. Let $C$ be a finite decomposition category. Let $\operatorname{Arr}(C)_{+}=\operatorname{Arr}(C) \backslash\left\{\mathrm{id}_{x}: x \in \mathrm{Ob}(C)\right\}$, and let $1_{A\langle\langle C\rangle\rangle}=\sum_{x \in \mathrm{Ob}(C)} \mathrm{id}_{x}$. Let us define

$$
\mathfrak{I}(A)=\left\{S \in A\langle\langle C\rangle\rangle: S=1_{A\langle\langle C\rangle\rangle}+\sum_{f \in \operatorname{Arr}(C)_{+}}\langle S \mid f\rangle f\right\} .
$$

Remark 9. We observe that in general for an arbitrary category $C, \mathfrak{J}(A)$ is not closed under $\circ$. Indeed, let us consider the finite category (actually, groupoid) $C$ with two objects $x, y$ and two nonidentity arrows $f: x \rightarrow y, g: y \rightarrow x$ with $f \circ g=\mathrm{id}_{y}$. It is obviously a finite decomposition category (since it is a finite category). Let $S=\mathrm{id}_{x}+\mathrm{id}_{y}-f$ and $T=\mathrm{id}_{x}+\mathrm{id}_{y}+g$. Then, $S T=\mathrm{id}_{x}+g-f \notin \mathfrak{J}(A)$. This cannot happen anymore if $C$ is locally finite (because no nonidentity arrows may be left or right invertible in such categories).

Lemma 10. Let one assumes that $C$ is a locally finite category. Then, $\mathfrak{\Im}(A)$ is a group under multiplication with identity $1_{A\langle\langle C\rangle\rangle}$. Actually, $\mathfrak{I}$ is an R-group.

Proof. Let us first check that $\mathfrak{\Im}(A)$ is a monoid. Let $S, T \in$ $\mathfrak{I}(A)$. Because $C$ is locally finite, we have for every $x \in \mathrm{Ob}(C)$, $\left\langle S T \mid \mathrm{id}_{x}\right\rangle=\left\langle S \mid \mathrm{id}_{x}\right\rangle\left\langle T \mid \mathrm{id}_{x}\right\rangle=1_{A}$ so that $S T \in \mathfrak{I}(A)$. Moreover, it is clear that $1_{A\langle\langle C\rangle\rangle}$ is a two-sided identity of $\mathfrak{I}(A)$ which turns to be a monoid. Now, let $S \in \mathfrak{J}(A)$. Let us define a collection of scalars $\left(T_{f}\right)_{f \in \operatorname{Arr}(C)}$ by induction on the length of $f$. First, $T_{\mathrm{id}_{x}}=1_{A}$ for every $x \in \mathrm{Ob}(C)$. Let $f \in \operatorname{Arr}(C)$ with $\ell(f)=1$. Then, we define $T_{f}=$ $-\langle S \mid f\rangle$. By induction, we define for $f$ with $\ell(f)=n+1$, $T_{f}=-\sum_{g h=f ; g \neq \operatorname{id}_{\partial_{1}(f)}}\langle S \mid g\rangle T_{h}$. Let us define $T=\sum_{f} T_{f} f$. Then, $T \in \mathfrak{J}(A)$, and $S T=1_{A\langle\langle C\rangle\rangle}$ (as $T$ is built to satisfy this property). By the same reasoning, $T$ admits itself a right inverse say $S^{\prime} \in \mathfrak{\Im}(A)$. Then, $S=S 1_{A\langle\langle C\rangle\rangle}=S\left(T S^{\prime}\right)=S^{\prime}$ so that $S$ admits $T$ as a two-sided inverse. The fact that $\mathfrak{I}$ is an $R$-group is obvious.

Remark 11. We observe that if we consider any locally finite monoid $M$ as a category with only one object, then $\mathfrak{\Im}(A)$ is the group of invertible series of the form $1+T$ (where $T$ is a series without constant terms) in the large monoid algebra $A[[M]]$ (see, e.g., [20]). In particular, if $M$ is $\mathbb{N}$, then $\mathfrak{I}$ is the $R$-group $G$ of invertible series of Example 2.

For the remainder of this subsection, $C$ is assumed to be a locally finite category. The set $\operatorname{Arr}(C)_{+}=\bigcup_{\ell \geq 1} \operatorname{Arr}(C)_{\ell}$ obviously forms the coordinate system of $\mathfrak{\Im}$ (see Section 3 ). Consequently, the polynomial algebra $R\left[\operatorname{Arr}(C)_{+}\right]$is the coordinate Hopf algebra that represents $\mathfrak{\Im}$. Again as in Section 3, its coproduct $\Delta$ is computed through the product in $\mathfrak{\Im}\left(R\left[\operatorname{Arr}(C)_{+}\right] \otimes_{R} R\left[\operatorname{Arr}(C)_{+}\right]\right)$of the series $S=1_{A\langle\langle C\rangle\rangle}+$ $\sum_{f \in \operatorname{Arr}(C)_{+}}(f \otimes 1) f$ and $T=1_{A\langle\langle C\rangle\rangle}+\sum_{f \in \operatorname{Arr}(C)_{+}}(1 \otimes f) f$ (where $\left.A=R\left[\operatorname{Arr}(C)_{+}\right] \otimes_{R} R\left[\operatorname{Arr}(C)_{+}\right]\right)$. Thus, $\Delta=\sum_{f \in \operatorname{Arr}(C)_{+}}(f \otimes$ $\left.1+1 \otimes f+\sum_{g \circ h=f}(g \otimes h)\right) f$, and consequently, $\Delta(f)=$ $f \otimes 1+1 \otimes f+\sum_{g \circ h=f}(g \otimes h)$ for every $f \in \operatorname{Arr}(C)_{+}$. The counit is given by $\epsilon(f)=0$ for every $f \in \operatorname{Arr}(C)_{+}$. To determine its antipode $s$, we need to compute the inverse of the series $S=1+\sum_{f \in \operatorname{Arr}(C)_{+}} f f \in \mathfrak{I}\left(R\left[\operatorname{Arr}(C)_{+}\right]\right)$. By recurrence on the length of $f$, we thus obtain that $s(f)=-f-\sum_{(g, h) \in D_{2}(f)} g s(h)$ for every $f \in f \in \operatorname{Arr}(C)_{+}$.

Remark 12. As in Remark 11, let us consider a locally finite monoid $M$ seen as a one-object category. In this case the set $M^{+}$of nonidentity elements of $M$ is the coordinate system of $\mathfrak{I}$. The coproduct on $R\left[M^{+}\right]$is then given by $\Delta(x)=x \otimes 1+$ $1 \otimes x+\sum_{y z=x ; y, z \in M^{+}} y \otimes z$. In particular, for $M=\mathbb{N}$, then $R\left[\mathbb{N}^{+}\right]$is similar to the usual divided power Hopf algebra (see for instance [5]).

6.2. Group of (Unipotent) Reversible Series. Let $A$ be a commutative $R$-algebra with a unit. Let $C$ be a finite semicategory. From Section 5, the free category $\underline{C}^{*}$ generated by 
the underlying graph $\underline{C}$ of $C$ is a locally finite category, and by Lemma $8, \mathfrak{M}(A)=\left\{S \in \mathfrak{M}_{\geq 1}: \forall f \in \operatorname{Arr}(C),\langle S\right.$ | $(f)\rangle \neq 0\}=\left\{S \in R\left\langle\left\langle\underline{C}^{*}\right\rangle\right\rangle: \forall x \in \mathrm{Ob}(C),\left\langle S \mid \mathrm{id}_{x}\right\rangle=\right.$ $0, \forall f \in \operatorname{Arr}(C),\langle S \mid(f)\rangle \neq 0\}$ is a group under substitution $\circ$, when $A$ is a field. We consider $\mathfrak{M}_{1}(A)=\left\{S \in R\left\langle\left\langle\underline{C}^{*}\right\rangle\right\rangle\right.$ : $\left.\left.\forall x \in \mathrm{Ob}(C),\left\langle S \mid \mathrm{id}_{x}\right\rangle=0, \forall f \in \operatorname{Arr}(C),\langle S| \overline{(f}\right)\right\rangle=$ $\left.1_{A}\right\} \subseteq \mathfrak{M}(A)$. Therefore, $S \in \mathfrak{M}_{1}(A)$ if, and only if, there exists $T \in \mathfrak{M}_{\geq 2}(A)$ such that $S=I+T$, where we recall that $I=$ $\sum_{f \in \operatorname{Arr}(C)}(f)$ and $\mathfrak{M}_{\geq n}(A)=\left\{S \in R\left\langle\left\langle\underline{C}^{*}\right\rangle\right\rangle: \omega(S) \geq n\right\}$ (see Section 4). According to the point (3) of Lemma 8, $\mathfrak{M}_{1}(A)$ is closed under inversion, and since $\langle S \circ T \mid(f)\rangle=\langle S|$ $(f)\rangle\left\langle T_{f} \mid(f)\right\rangle=\left\langle T_{f} \mid(f)\right\rangle=\left\langle\sum_{\vec{f}, \pi(\vec{f})=f}\langle T \mid \vec{f}\rangle \vec{f} \mid f\right\rangle=$ $\langle T \mid(f)\rangle=1, \mathfrak{M}_{1}(A)$ forms a monoid under $\circ$, and thus a group. It is clearly an $R$-group.

Remark 13. When $C$ is the semicategory with one object and one arrow as in Example 7, then $\mathfrak{M}_{1}(A)$ is the group of reversible formal power series in one variable of the form $x+\mathcal{O}\left(x^{2}\right)$.

The set $\operatorname{Arr}\left(\underline{C}^{*}\right)_{\geq 2}=\bigcup_{\ell \geq 2} \operatorname{Arr}\left(\underline{C}^{*}\right)_{\ell}$, where we recall that $\operatorname{Arr}\left(\underline{C}^{*}\right)_{\ell}$ is the set of all arrows of $\underline{C}^{*}$ of length $\ell$, is a coordinate system of the group $\bar{M}_{1}(A)$. Let us determine the coproduct $\Delta$, counit $\epsilon$, and antipode $s$ of the coordinate algebra $R\left[\operatorname{Arr}\left(\underline{C}^{*}\right)_{\geq 2}\right]$. Let $S=I+\sum_{\vec{f}}(\vec{f} \otimes 1) \vec{f}$ and $T=I+\sum_{\vec{f}}(1 \otimes \vec{f}) \vec{f}$ be two series in $\mathfrak{M}_{1}\left(R\left[\operatorname{Arr}\left(\underline{C}^{*}\right)_{\geq 2}\right] \otimes_{R} R\left[\operatorname{Arr}\left(\underline{C}^{*}\right)_{\geq 2}\right]\right)$. According to Lemma 6, $\left\langle S \circ T\left(f_{1}, \ldots, f_{n}\right)\right\rangle=$ $\left(f_{1}, \ldots, f_{n}\right) \otimes 1+1 \otimes\left(f_{1}, \ldots, f_{n}\right)+$ $\sum_{\ell=2}^{n-1} \sum_{\left(\vec{f}^{(1)}, \ldots, \vec{f}^{(\ell)}\right) \in D_{\ell}\left(f_{1}, \ldots, f_{n}\right)}\left(\pi\left(\vec{f}^{(1)}\right), \ldots, \pi\left(\vec{f}^{(\ell)}\right)\right) \otimes \vec{f}^{(1)} \ldots \vec{f}^{(\ell)}$ for every $n \geq 2$, and every arrow $\left(f_{1}, \ldots, f_{n}\right)$. It amounts to say that $\Delta\left(\left(f_{1}, \ldots, f_{n}\right)\right)=\left(f_{1}, \ldots, f_{n}\right) \otimes 1+1 \otimes\left(f_{1}, \ldots, f_{n}\right)+$ $\sum_{\ell=2}^{n-1} \sum_{\left(\vec{f}^{(1)}, \ldots, \vec{f}^{(\ell)}\right) \in D_{\ell}\left(f_{1}, \ldots, f_{n}\right)}\left(\pi\left(\vec{f}^{(1)}\right), \ldots, \pi\left(\vec{f}^{(\ell)}\right)\right) \otimes \vec{f}^{(1)} \ldots \vec{f}^{(\ell)}$. As usual the counit is given by $\epsilon(\vec{f})=0$ for every $\vec{f} \in \operatorname{Arr}\left(\underline{C}^{*}\right)_{\geq 2}$. It remains to determine the antipode $s$ from the inverse series of $I+\sum_{\vec{f}} \vec{f} \vec{f} \in \mathfrak{M}_{1}\left(R\left[\operatorname{Arr}\left(\underline{C}^{*}\right)_{\geq 2}\right]\right)$. It is easily seen to be given by

$$
\begin{aligned}
s( & \left.\left(f_{1}, \ldots, f_{n}\right)\right) \\
= & -\left(f_{1}, \ldots, f_{n}\right) \\
& -\sum_{\ell=2}^{n-1} \sum_{\left.\vec{f}^{(1)}, \ldots, \vec{f}^{(\ell)}\right) \in D_{\ell}\left(f_{1}, \ldots, f_{n}\right)}\left(\pi\left(\vec{f}^{(1)}\right), \ldots, \pi\left(\vec{f}^{(\ell)}\right)\right) \\
& \times s\left(\vec{f}^{(1)}\right) \ldots s\left(\vec{f}^{(\ell)}\right),
\end{aligned}
$$

for every arrow $\left(f_{1}, \ldots, f_{n}\right), n \geq 2$.

Remark 14. As in Remark 13, if $C$ is the semicategory with a single object and a single arrow, then the coordinate Hopf algebra of $\mathfrak{M}_{1}$ is the usual Faà di Bruno Hopf algebra (see $[11,15])$.

6.3. Interaction between both Hopf Algebras. The notion of smash coproduct may be found in [28-30], for instance.
Let $\mathbb{K}$ be a field. Let $H$ and $C$ be two Hopf algebras over $\mathbb{K}$. According to [29], if $H$ is commutative and $C$ is an $H$ comodule bialgebra, then the tensor algebra $C \otimes_{\mathbb{K}} H$ admits a structure of Hopf algebra, called the smash coproduct, and is denoted by $C \# H$. An affine group on a field $\mathbb{K}$ will be referred to as a proaffine algebraic group.

Example 15 (see $[29,30]$ ). Let $N$ and $K$ be two proaffine algebraic groups, represented by the coordinate Hopf algebras $\mathcal{O}(N)$ and $\mathcal{O}(K)$. Let us assume that we have a semidirect product $N \rtimes_{\tau} K$ of proaffine algebraic groups, that is, $\tau: K \times N \rightarrow N$ is a natural transformation that induces a group action from $K$ on $N$ by proaffine algebraic group automorphisms. Then, according to Yoneda's lemma, $\tau$ induces an algebra map $\mathcal{O}(N) \rightarrow \mathcal{O}(K) \otimes_{\mathbb{K}} \mathcal{O}(N)$ that turns $\mathscr{O}(N)$ into a $\mathscr{O}(K)$-comodule bialgebra. Thus, $\mathcal{O}(N) \# \mathscr{O}(K)$ is a Hopf algebra that can be shown to be isomorphic to $\mathcal{O}\left(N \rtimes_{\tau} K\right)$.

Example 15 may be applied on the two affine groups of series over a category previously introduced. Let $R$ be a commutative ring with a unit and let $C$ be a finite semicategory. We consider the group-valued functors of series $\mathfrak{J}$ and $\mathfrak{M}_{1}$ over the locally finite category $\underline{C}^{*}$ so that the results of Sections 6.1 and 6.2 hold. Let $A$ be any commutative $R$ algebra with a unit. For every $S \in \mathfrak{J}(A)$ and $T \in \mathfrak{M}_{1}(A)$, $S \circ T \in \mathfrak{I}(A)$ (by Lemma 6). Moreover, $\rho_{T}: \mathfrak{I} \rightarrow \mathfrak{I}(A)$ is invertible with inverse $\rho_{T^{\circ-1}}$. Actually, it defines a group antihomomorphism $\rho: \mathfrak{M}_{1}(A) \rightarrow \operatorname{Aut}(\mathfrak{J}(A))$ by $\rho(T)=$ $\rho_{T}$. Therefore, we obtain a structure of semidirect product $\mathfrak{I}(A) \rtimes \mathfrak{M}_{1}^{\mathrm{op}}(A)$ on $\mathfrak{I}(A) \times \mathfrak{M}_{1}(A)$ by $\left(S_{1}, T_{1}\right) \rtimes\left(S_{2}, T_{2}\right)=$ $\left(S_{1}\left(S_{2} \circ T_{1}\right), T_{2} \circ T_{1}\right)$ and with $\left(1_{\mathbb{K}\left\langle\left\langle\underline{C}^{*}\right\rangle\right\rangle}, I\right)$ as identity (where $G^{\text {op }}$ denotes the opposite group of a group $\left.G\right)$. The inverse of $(S, T)$ is given by $\left(S^{-1} \circ T^{\circ-1}, T^{\circ-1}\right)$. This construction is easily seen to be natural in $A$ so that we have a semidirect $R$-group functor $\mathfrak{I} \rtimes \mathfrak{M}_{1}^{\mathrm{op}}$.

Remark 16. This situation generalizes the case of formal power series in one-variable $x$ where we have a semidirect product $G \rtimes H$ (using the notations from Example 2) given by exactly the same formula $\left(S_{1}, T_{1}\right) \rtimes\left(S_{2}, T_{2}\right)=$ $\left(S_{1}\left(S_{2} \circ T_{1}\right), T_{2} \circ T_{1}\right)$ and with $(1, x)$ as unit element. See for instance [31].

Now, let us assume that $R$ is a field $\mathbb{K}$ (in order to apply the results from $[29,30])$. According to Example 15, we have a structure of Hopf algebra given by the smash coproduct $\mathcal{O}(\mathfrak{I}) \# \mathcal{O}\left(\mathfrak{M}_{1}^{\mathrm{op}}\right) \cong \mathcal{O}\left(\mathfrak{I} \rtimes \mathfrak{M}_{1}^{\mathrm{op}}\right)$. It is clear that $\mathcal{O}\left(\mathfrak{M}_{1}^{\mathrm{op}}\right)$ is isomorphic to the Hopf algebra $\mathbb{K}\left[\operatorname{Arr}\left(\underline{C}^{*}\right)_{\geq 2}\right]^{\mathrm{op}}$ (which is the algebra $\mathbb{K}\left[\operatorname{Arr}\left(\underline{C}^{*}\right)_{\geq 2}\right]$ with opposite coproduct $\Delta^{\mathrm{op}}=\tau \circ \Delta$, where $\tau: \mathbb{K}\left[\operatorname{Arr}\left(\underline{C}^{*}\right)_{\geq 2}\right] \otimes_{\mathbb{K}} \mathbb{K}\left[\operatorname{Arr}\left(\underline{C}^{*}\right)_{\geq 2}\right] \rightarrow$ $\mathbb{K}\left[\operatorname{Arr}\left(\underline{C}^{*}\right)_{\geq 2}\right] \otimes_{\mathbb{K}} \mathbb{K}\left[\operatorname{Arr}\left(\underline{C}^{*}\right)_{\geq 2}\right]$ is the usual flip). Therefore, $\mathcal{O}\left(\mathfrak{I} \rtimes \overline{\mathfrak{M}}_{1}^{\mathrm{op}}\right) \cong \mathbb{K}\left[\operatorname{Arr}\left(\underline{C}^{*}\right)_{+}\right] \# \mathbb{K}\left[\operatorname{Arr}\left(\underline{C}^{*}\right)_{\geq 2}\right]^{\mathrm{op}}$. Moreover, a set-valued functor $\mathfrak{\Im} \rtimes \mathfrak{M}_{1}^{\mathrm{op}}$ is isomorphic to $\mathfrak{\Im} \times \mathfrak{M}_{1}^{\mathrm{op}}$ so that its coordinate algebra is $\mathbb{K}\left[\operatorname{Arr}\left(\underline{C}^{*}\right)_{+} \sqcup \operatorname{Arr}\left(\underline{C}^{*}\right)_{\geq 2}\right]$ (thus Theorem 1 is proved).

Remark 17. In the paper [32], the authors described a similar interaction between two Hopf algebras of functions on the so-called B-series [33, 34]. 


\section{Acknowledgments}

In the first version of this contribution, many results were obtained by long and complicated proofs involving (finite) duality (à la Sweedler). Moreover, the base ring was always assumed to be a field. We wish to thank the referees for their helpful comments and remarks. Proofs became more clear and easy using Yoneda's lemma as suggested by one of the referees who also explained that most of the results hold true on any commutative ring so that our original assumption on the base ring was removed.

\section{References}

[1] S. Warner, Topological Fields, vol. 157 of North-Holland Mathematical Studies, Elsevier, New York, NY, USA, 1989.

[2] J. Berstel and C. Reutenauer, Noncommutative Rational Series with Applications, vol. 137 of Encyclopedia of Mathematics and Its Applications, Cambridge University Press, Cambridge, UK, 2010.

[3] L. Poinsot, Contributions à l'Algèbre, à l'Analyse et à la Combinatoire des Endomorphismes sur les Espaces de Séries, (French Language), Habilitation à Diriger des Recherches en Mathématiques, Université Paris 13, Sorbonne Paris Cité, Villetaneuse, France, 2011, http://lipn.univ-paris13.fr/ poinsot/ HDR/HDR.pdf.

[4] L. Poinsot, "Rigidity of the topoogical dual of spaces of formal series with respect to product topologies," 2010, preprint, http://lipn.univ-paris13.fr/ poinsot/Articles/Top-dual-spacev8.pdf.

[5] S. Dascalescu, C. Nastasescu, and S. Raianu, Hopf Algebras, vol. 235 of Pure and Applied Mathematics, Marcel Dekker, New York, NY, USA, 2001.

[6] C. Kassel, Quantum Groups, vol. 155 of Graduate Texts in Mathematics, Springer, Berlin, Germany, 2010.

[7] M. Demazure and P. Gabriel, Groupes Algébriques: Volume 1, Elsevier, New York, NY, USA, 1970.

[8] G. Hochschild and G. D. Mostow, "Pro-affine algebraic groups," American Journal of Mathematics, vol. 91, no. 4, pp. 1127-1140, 1969.

[9] J. S. Milne, Basic Theory of Affine Group Schemes, Version 1. 00, On-line course material, 2012.

[10] S. MacLane, Categories for the Working Mathematician, vol. 5 of Graduate Texts in Mathematics, Springer, Berlin, Germany, 1998.

[11] H. Figueroa, J. M. Gracia-Bondía, and J. C. Várilly, "Faà di Bruno Hopf algebras," in Encyclopedia of Mathematics, M. Hazewinkel, Ed., Springer, Berlin, Germany.

[12] S. A. Joni and G. Rota, "Coalgebras and bialgebras in combinatorics," Studies in Applied Mathematics, vol. 61, pp. 93-139, 1979.

[13] W. R. Schmitt, "Incidence Hopf algebras," Journal of Pure and Applied Algebra, vol. 96, no. 3, pp. 299-330, 1994.

[14] S. A. Jennings, "Substitution groups of formal power series," Canadian Journal of Mathematics, vol. 6, pp. 325-340, 1954.

[15] L. Foissy, "Faà di Bruno subalgebras of the Hopf algebra of planar trees from combinatorial Dyson-Schwinger equations," Advances in Mathematics, vol. 218, no. 1, pp. 136-162, 2008.

[16] R. P. Stanley, Enumerative Combinatorics, Cambridge University Press, Cambridge, UK, 1997.
[17] P. Ageron, "Logic without self-deductibility, logical universalis," in Towards a General Theory of Logic, J. Y. Beziau, Ed., pp. 89-95, Birkhaüser, Basel, Switzerland, 2005.

[18] N. Bourbaki, Elements of Mathematics-Algebra, chapter 1-3, Springer, Berlin, Germany, 1989.

[19] A. H. Clifford and G. B. Preston, The Algebraic Theory of Semigroups, vol. 7 of Mathematical Surveys, American Mathematical Society, Providence, RI, USA, 1961.

[20] L. Poinsot, G. H. E. Duchamp, and C. Tollu, "Möbius inversion formula for monoids with zero," Semigroup Forum, vol. 81, no. 3, pp. 446-460, 2010.

[21] M. Content, F. Lemay, and P. Leroux, "Catégories de Möbius et fonctorialités : un cadre général pour l'inversion de Möbius," Journal of Combinatorial Theory A, vol. 28, pp. 169-190, 1980.

[22] P. Leroux, "Les catégories de Möbius," Cahiers de Topologie et Géométrie Différentielle, vol. 16, pp. 280-282, 1975.

[23] F. W. Lawvere and M. Menni, “The Hopf algebra of Möbius intervals," Theory and Applications of Categories, vol. 24, pp. 221$265,2010$.

[24] I. Assem, D. Simon, and A. Skowronski, Elements of the Representation Theory of Associative Algebras, 1: Techniques of Representation Theory, Cambridge University Press, Cambridge, UK, 2006.

[25] B. Mitchell, "Rings with several objects," Advances in Mathematics, vol. 8, no. 1, pp. 1-161, 1972.

[26] N. Bourbaki, Elements of Mathematics-General Topology, chapter 1-4, Springer, Berlin, Germany, 1998.

[27] L. Comtet, Advanced Combinatorics-The Art of Finite and infinite Expansions, Reidel, Dordrecht, The Netherlands, 1974.

[28] R. G. Heyneman and M. E. Sweedler, "Affine Hopf algebras, I," Journal of Algebra, vol. 13, no. 2, pp. 192-241, 1969.

[29] R. K. Molnar, "Semi-direct products of Hopf algebras," Journal of Algebra, vol. 47, no. 1, pp. 29-51, 1977.

[30] D. E. Radford, "The structure of Hopf algebras with a projection," Journal of Algebra, vol. 92, no. 2, pp. 322-347, 1985.

[31] L. Poinsot and G. H. E. Duchamp, "A formal calculus on the Riordan near algebra," Advances and Applications in Discrete Mathematics, vol. 6, no. 1, pp. 11-44, 2010.

[32] D. Calaque, K. Ebrahimi-Fard, and D. Manchon, “Two interacting Hopf algebras of trees: a Hopf-algebraic approach to composition and substitution of B-series," Advances in Applied Mathematics, vol. 47, no. 2, pp. 282-308, 2011.

[33] Ph. Chartier, E. Hairer, and G. Vilmart, "A substitution law for B-series vector fields," Preprint INRIA 5498, INRIA, Paris, France, 2005.

[34] E. Hairer, C. Lubich, and G. Wanner, Geometric Numerical Integration Structure-Preserving Algorithms for Ordinary Differential Equations, vol. 31 of Springer Series in Computational Mathematics, Springer, Berlin, Germany, 2002. 


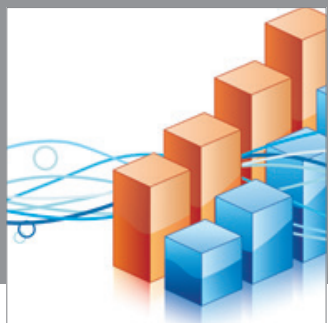

Advances in

Operations Research

mansans

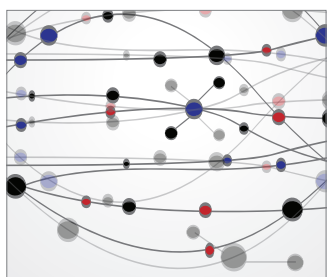

The Scientific World Journal
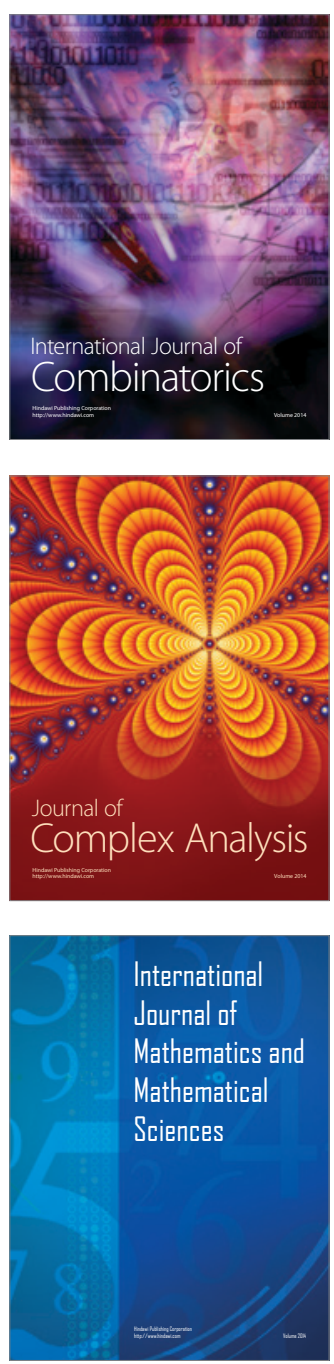
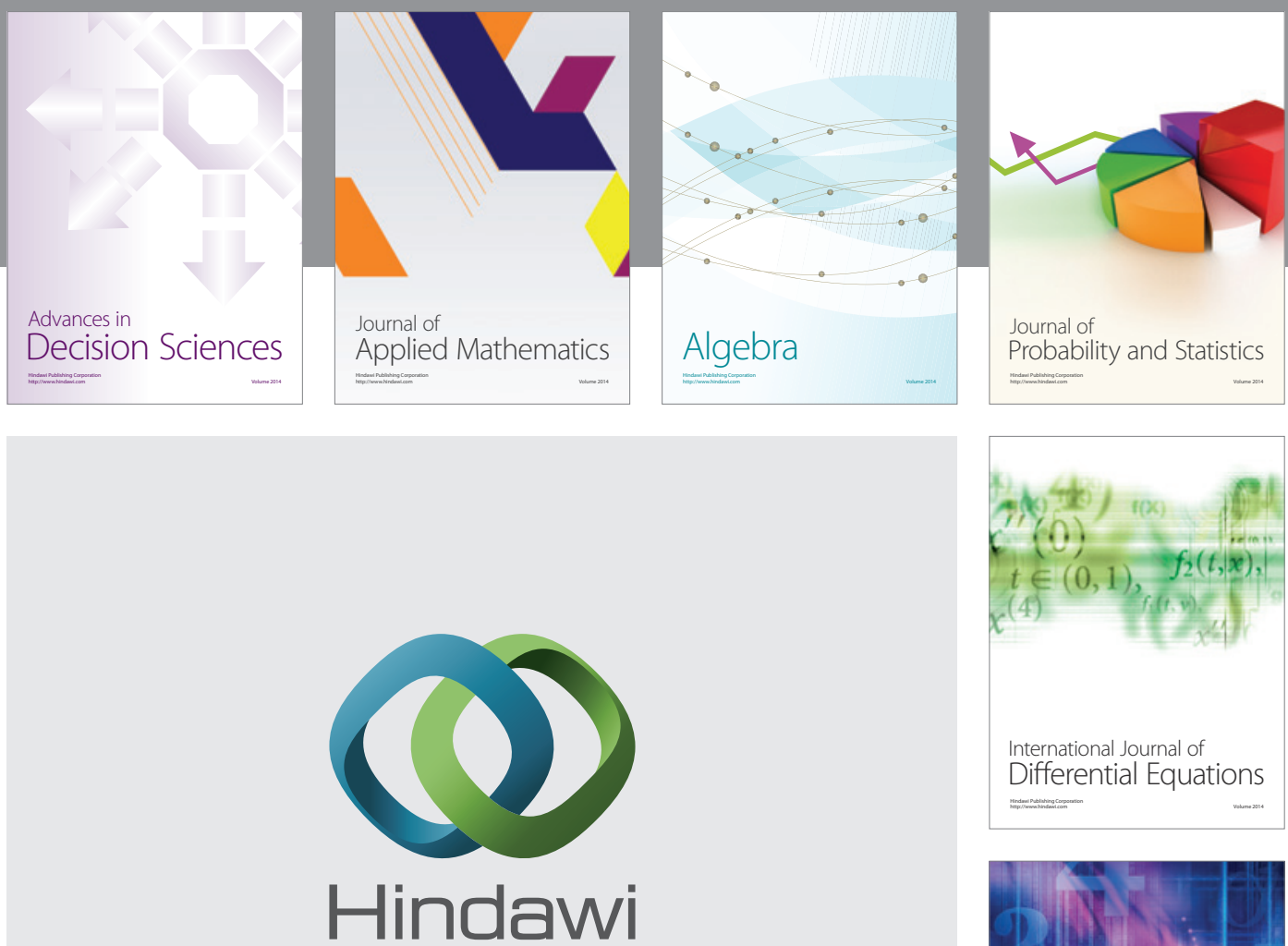

Submit your manuscripts at http://www.hindawi.com
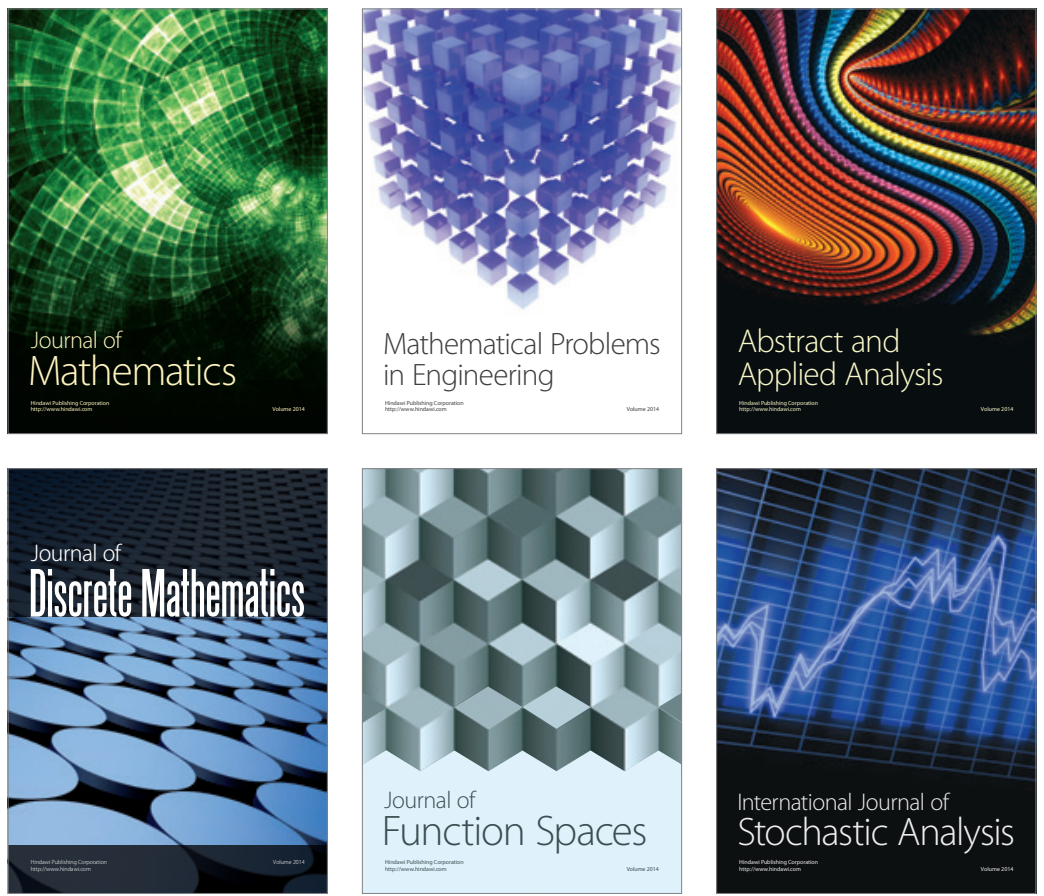

Journal of

Function Spaces

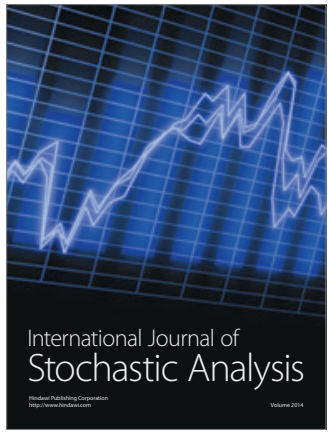

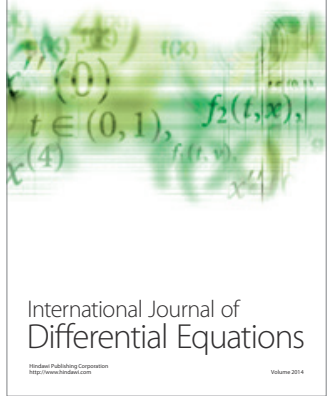
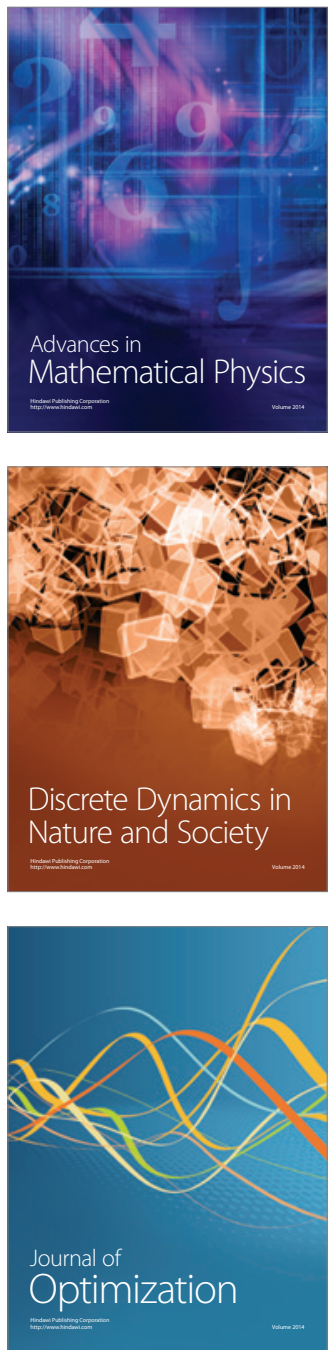